\title{
Comentários sobre a interdisciplinaridade da física e físico-química
}

\author{
Some comments on the \\ relationship between Physics and \\ Physical Chemistry
}

Uma primeira versão deste artigo, intitulada

'A física e a interdisciplinaridade: físico-química', foi apresentada em palestra proferida

pelas autoras em 13 de outubro de 1999, no

Museu de Astronomia e

Ciências Afins (Rio de Janeiro), integrando a série

Mast Colloquia-Memória da Física

Silvia Tiomno Tolmasquim

\author{
Professora titular aposentada do Departamento de Físico-Química, Instituto de Química, UFRJ \\ Rua Rodolfo Dantas, 16/604 \\ 22020-040 Rio de Janeiro — RJ Brasil \\ silviatt@esquadro.com.br \\ Bartyra de Castro Arezzo \\ Pesquisadora titular aposentada da CNEN e ex-professora adjunta do Departamento \\ de Físico-Química, Instituto de Química, UFRJ \\ Rua General Góis Monteiro, 8 bl. F/1504 \\ 22290-080 Rio de Janeiro - RJ Brasil \\ bartyra@openlink.com.br
}

\begin{abstract}
Além de apresentar comentários sobre as fronteiras entre a física e a físico-química, o trabalho procura ilustrar a interdisciplinaridade dessas duas ciências considerando o histórico profissional de alguns físicos e físico-químicos que desenvolveram atividades nas décadas de 1920 a 1980. Destaca, ainda, o papel desempenhado pela extinta Faculdade Nacional de Filosofia da Universidade do Brasil como forte elemento de integração entre estudantes e professores das diferentes áreas do conhecimento, no Rio de Janeiro.

PALAVRAS-CHAVE: história da físico-química, biografias, universidades no Brasil, institutos de pesquisa no Brasil.

Besides commenting on the boundaries between physics and physical chemistry, this article tries to depict the relationship between these two sciences by considering the professional history of some physicists and physical chemists from 1920 to 1980. It also focus on the role played by the extinct Faculdade Nacional de Filosofia da Universidade do Brasil as a strong integration element for students and professors from different fields of studies in Rio de Janeiro. KEYWORDS: history of physical chemistry, biographies, universities in Brazil, research institutes in Brazil.
\end{abstract}


A físico-química pode ser definida didaticamente como a ciência que visa esclarecer os princípios que regulam as transformações da matéria, conhecidas como reações químicas. Para isso, estuda as propriedades físicas das substâncias, bem como a influência de fatores tais como temperatura, pressão, concentração, eletricidade e luz na transformação considerada. Com um método de trabalho semelhante ao da física, procura coletar dados que definam as propriedades do sistema, correlacioná-los por meio de palavras, gráficos ou equações matemáticas e, finalmente, transformá-los em leis e dar-lhes fundamento teórico. Com o aperfeiçoamento das medidas experimentais e o maior uso da matemática, a físico-química vem-se tornando cada vez mais exata (Daniels et al., 1958, pp. 1-3).

De forma geral, a físico-química compreende a termodinâmica, ou seja, a investigação das trocas energéticas, o que torna possível prever o quanto uma dada reação avança até que o equilíbrio seja atingido; a cinética química, que envolve o fator tempo e estuda os processos e mecanismos de reação; a eletroquímica, que trata dos eletrólitos e do equilibrio em soluções eletrolíticas; o estudo da estrutura das moléculas, feito sobretudo pela investigação dos espectros moleculares e atômicos, da difração de raios X e da difração de elétrons.

Desde que eclodiu como um ramo separado da ciência, a físicoquímica vem alargando seu campo de atuação com as novas descobertas, justificando resultados experimentais em desacordo com previsões teóricas da física ou da química. Entre as novas áreas abrangidas, podem-se citar, entre outras, a química dos fenômenos de superfície (como a adsorsão e a coloidoquímica), a radioquímica e a química nuclear. Colocando-se entre a física e a química, que são áreas muito próximas e continuamente inter-relacionadas, há inúmeras questões que podem ser tratadas tanto pela físico-química como pela física, da mesma forma que outras podem ser abordadas pela físico-química ou pela química.

Horacio Macedo (1976, p. 160) sintetiza a interdisciplinaridade de física e físico-química com as seguintes palavras:

A físico-química desenvolveu-se a partir dos fins do século XIX, graças aos trabalhos de Ostwald sobre eletroquímica. A sua evolução foi, no início, predominantemente marcada pela evolução da química de que era fortemente subsidiária (em alguns textos ainda se usa a denominação químico-física, para assinalar esta dependência). A rápida expansão da física e a diversificação do seu domínio mostraram a necessidade de uma ciência de fronteira em que tanto a física como a química tivessem importância e contribuíssem simultaneamente para a resolução de seus problemas. Atualmente, é flagrante a predominância dos processos físicos não só na investigação experimental, como na análise teórica dos fenômenos físicoquímicos. 


\section{Físicos e físico-químicos}

Além de ponderações sobre os temas abrangidos e os métodos de trabalho dessas ciências, a interdisciplinaridade da física e físico-química pode ser ilustrada, pelo menos no Rio de Janeiro, considerando-se o histórico profissional de algumas pessoas que atuaram nas duas áreas nas décadas de 1920 a 1980. Há vários cientistas, alguns ainda em atividade, cujos nomes situam-se bem tanto em uma como em outra disciplina.

Joaquim da Costa Ribeiro (1906-60) cita, junto aos físicos, vários físico-químicos, e dedica, por exemplo, a Carneiro Felippe (1886-1951), do Instituto Oswaldo Cruz (IOC), pioneiro da físico-química no Rio de Janeiro, as seguintes palavras:

Uma referência muito especial deve ser feita à singular figura do professor José Carneiro Felipe que, embora tendo-se dedicado mais especificamente à química e à físico-química, exerceu, graças à sua profunda e multiforme cultura científica, poderosa influência sobre um grande número de jovens pesquisadores brasileiros, inclusive no campo da física (Costa Ribeiro, 1994, p. 198).

Já Shozo Motoyama (1979, p. 70) assim se expressa em relação a Carneiro Felippe e Henrique Morize (1860-1930), professor de física da Escola Politécnica do Rio de Janeiro: "Graças a esses e outros idealistas, os temas de grande atualidade na época, como descarga elétrica em gases rarefeitos, raios $\mathrm{X}$, radioatividade etc., tornaram-se conhecidos. Iniciava-se destarte a formação de um ambiente salutar mais propício à física."

Carneiro Felippe, engenheiro formado pela Escola de Minas de Ouro Preto, foi diretor (1918-19) do Laboratório Químico e Biológico do Estado de Minas Gerais. Ingressou posteriormente, a convite de Carlos Chagas, no IOC, onde instalou o Departamento de FísicoQuímica. Formou-se ali a primeira geração de físico-químicos do Rio de Janeiro, como João Christovão Cardoso, Augusto Zamith e Carlos Chagas Filho, que mais tarde assumiu a liderança na área da biofísica. No Rio de Janeiro, Carneiro Felippe lecionou físico-química no curso de química industrial, anexo à Escola Politécnica, na Escola Nacional de Química e no Curso de Aplicação do IOC, onde também ensinou biometria com aplicação de estatística matemática. Foi ainda assistentetécnico de ensino do Ministério da Educação e representou o Brasil em reuniões internacionais sobre estatística. Participou de várias entidades científicas, foi membro titular da Academia Brasileira de Ciências, integrou o Conselho Nacional de Educação, a Comissão de Reajustamento do Funcionalismo Público Civil, a Comissão de Estudos da Cidade Universitária e do Conselho Nacional de Pesquisas (CNPq), para cuja presidência fora designado no dia em que faleceu. 


\begin{abstract}
Nos domínios da físico-química devem ser mencionadas, ainda, as contribuições originais de pesquisadores isolados, destacando-se os trabalhos de Roberto Betim Paes Leme, ${ }^{1}$ no Museu Nacional, sobre um novo método de análise espectral quantitativa, de E. L. da Fonseca Costa, no Instituto Nacional de Tecnologia, sobre a físico-química das ligas ferro-carbono, sobre a cinemática do sistema ferro-oxigênio-carbono e especialmente sobre um novo e original método de determinação das velocidades de reação em função da temperatura, utilizando uma termobalança de sua invenção, e os numerosos trabalhos de Alvaro Alberto sobre a físico-química dos explosivos e assuntos correlatos, entre os quais devem ser sobretudo mencionados seus estudos sobre os critérios da estabilidade química, sobre o controle da estabilidade dos cristais e seus calores de formação e de solução, sobre as reações em fase sólida etc. (Costa Ribeiro, 1994, p. 200).
\end{abstract}

Foram os esforços pessoais de Alvaro Alberto da Mota e Silva, almirante, matemático e físico-químico, que tornaram possível a criação do Centro Brasileiro de Pesquisas Físicas (CBPF), em 1949, e do CNPq, em 1951. "A principal preocupação de Alvaro Alberto e a principal função destas instituições foi dar o ponto de partida para a pesquisa nuclear no Brasil" (Schwartzman, 1979, p. 289).

O Instituto Nacional de Tecnologia (INT), fundado em 1921, por Ernesto Lopes da Fonseca Costa, como Estação Experimental de Combustíveis e Minérios, destacou-se como centro de pesquisas, onde foram desenvolvidos importantes trabalhos na área da química e da física. Neste último campo, merecem menção as investigações de Bernhard Gross e seus colaboradores. O INT não tinha um setor que trabalhasse especificamente em físico-química, porém seus técnicos usavam métodos físico-químicos de análise na solução de questões de química e de tecnologia ou ainda na investigação de estruturas moleculares de proteínas e de amidos. Sem dúvida, houve estudos como os de Fonseca Costa, fortemente baseados na físico-química. Citam-se também, entre outras, várias pesquisas nos campos da corrosão, adsorsão, catálise e propriedades tixotrópicas, realizadas tendo em vista aplicações em diversas áreas tecnológicas.

Costa Ribeiro (op. cit., p. 206) refere-se ao

Laboratório da Produção Mineral, onde no campo da físico-química trabalha o conhecido professor Hans Zocher. As pesquisas do professor H. Zocher têm versado sobre a assimetria dos cristais. No mesmo laboratório trabalhou também H. G. de Carvalho, tendo publicado trabalhos sobre o poder detentor (stopping power) das emulsões nucleares, sobre o alcance das partículas alfa na água e no gelo, e sobre várias questões de físicoquímica.

Hans E. W. Zocher (1893-1969), nascido na Alemanha, antigo professor da Universidade de Berlim e da Escola Técnica Superior de 
Praga, veio em 1946 para o Laboratório da Produção Mineral a convite do ilustre químico Fritz-Feigl (1891-1971), especialista em microanálise. Como técnico contratado, função que ocupou até sua morte, Zocher realizou pesquisas sobre diversos assuntos de físicoquímica, como colóides anisotrópicos, quimioluminescência, fotoquímica, mesofases, entre outros, tendo publicado mais de oitenta trabalhos. Seus estudos na área da coloidoquímica obtiveram repercussão internacional.

Hervasio Guimarães de Carvalho (1916-99), químico industrial pela Escola de Engenharia da Universidade do Recife, é um representante da interdisciplinaridade da física e físico-química. Em 1946, conquistou a cátedra de físico-química e eletroquímica na Escola de Engenharia. Em 1954, fez doutoramento em engenharia nuclear na North Caroline State University, passando a atuar nessa área. Trabalhou em várias instituições no exterior, destacando-se o Centro de Pesquisa Nuclear de Casaccia e o Instituto de Física Nuclear de Nápoles, onde foi professor visitante. No Brasil, trabalhou na Companhia de Pesquisa de Recursos Minerais (CPRM), na Universidade Federal do Rio de Janeiro (UFRJ), na Pontifícia Universidade Católica (PUC), no CBPF e na Comissão Nacional de Energia Nuclear (CNEN), que presidiu durante 13 anos (1969-82). Foi o primeiro presidente da Companhia Brasileira de Tecnologia Nuclear (CBTN) e da sua sucessora, a Nuclebrás. No CBPF, onde chefiou o Departamento de Física Experimental e, em seguida, o de Físico-Química (radioatividade), foi também diretor científico, diretor executivo e chefe do Departamento de Intercâmbio Cultural. Na UFRJ, recebeu os títulos de doutor em ciências (Escola Nacional de Química) e doutor em físico-química (Escola de Engenharia) e lecionou engenharia nuclear, físico-química, físico-química de metais e física. A maioria de seus trabalhos integra a área de física nuclear, mas há um número expressivo nos campos da físico-química e da química.

Poderíamos relacionar muitos professores e pesquisadores que tiveram sua primeira formação em cursos de química e depois suplementaram-na em cursos de física; ou que, especializados em físico-química, atuaram na interface da física com a físico-química. Parece-nos, porém, que os nomes mencionados a seguir ilustram suficientemente $\mathrm{O}$ assunto.

Sergio Pereira da Silva Porto (1926-79), bacharel e licenciado em química pela Faculdade Nacional de Filosofia (FNFi) da Universidade do Brasil (UB), hoje UFRJ, foi durante curto espaço de tempo auxiliar de ensino, inicialmente de química orgânica e em seguida de físicoquímica. Nesse período, exercia também a função de professor de química no Colégio Estadual, em Niterói. Obteve o grau de doutor em física na Johns Hopkins University, passando então a atuar nesse campo da ciência. Sergio Porto, pioneiro no estudo das aplicações do raio laser, especialmente no uso dessa fonte de radiação em espectroscopia Raman e em medicina, tornou-se um dos cientistas brasileiros mais 
famosos no exterior. Integram seu currículo a apresentação de cerca de quarenta trabalhos em sociedades profissionais e a de sessenta palestras por convites de entidades científicas de diversos países. Nos Estados Unidos, foi pesquisador e supervisor de pesquisas do Departamento de Eletrônica Quântica dos Bell Telephone Laboratories e professor de física e engenharia elétrica na University of Southern California. No Brasil, exerceu o magistério no Departamento de Física do Instituto Tecnológico da Aeronáutica (ITA), em São José dos Campos (SP), e no Instituto de Física Gleb Wathagin, na Universidade Estadual de Campinas (Unicamp). Na Unicamp, criou um grupo de eletrônica quântica e exerceu a coordenação geral dos institutos. Sergio Porto batalhou ativamente na promoção de oportunidades educacionais e de pesquisa nos Estados Unidos para estudantes latino-americanos de física e engenharia (Mooradian, 1979, p. VII).

Jacques A. Danon (1924-89) caracterizou-se pela multidisciplinaridade. Graduado pela Escola Nacional de Química (ENQ) da UB, foi em seguida admitido no Instituto do Rádio de Paris, dirigido por Irene Joliot-Curie, onde atuou no campo da físico-química e da química nuclear, especialmente na separação de radioelementos. De volta ao Brasil, lecionou físico-química na ENQ, integrando o grupo do professor catedrático Augusto Araujo Lopes Zamith. Mais tarde, chefiou o Departamento de Física Molecular e Estado Sólido do CBPF, onde foram desenvolvidos estudos nos campos da ressonância paramagnética eletrônica e do efeito Mössbauer em compostos inorgânicos e complexos metálicos. Foi pesquisador do Centro Nacional de Pesquisa Científica de Paris, do Centro de Pesquisa Nuclear da Universidade Livre da Bélgica, além de diretor do Observatório Nacional, no Rio de Janeiro. Seus trabalhos na área do efeito Mössbauer levaram-no a pesquisar certas ligas de ferro, bem como o ferro em sistemas orgânicos. Daí passou a realizar estudos sobre meteoritos metálicos e não-metálicos. Atuou ainda no campo da datação de artefatos líticos e cerâmicas arqueológicas. Jacques Danon não só produziu importantes trabalhos, mas também soube formar equipes de jovens cientistas que continuam desenvolvendo pesquisas sobre esses temas (Muniz, 1999, pp. 7-10).

José Israel Vargas, ${ }^{2}$ bacharel e licenciado em química pela Universidade Federal de Minas Gerais (UFMG), qualificou-se como doutor em química pela Universidade de Cambridge, Inglaterra. Exerceu o magistério na UFMG como professor catedrático de físico-química e química superior, tendo chefiado o Departamento de Química do Instituto de Ciências Exatas dessa universidade. No período de 1986 a 1995, foi pesquisador titular do CBPF, no Rio de Janeiro. Atuando em diferentes áreas de pesquisa, orientou a elaboração de teses de mestrado e doutorado no Brasil e na França e tem integrado e dirigido grupos de estudo no país e no exterior. Com uma centena de trabalhos científicos publicados nos domínios das conseqüências físico-químicas de 
transformações nucleares, química do estado sólido e interações hiperfinas nos sólidos, política científica, política de energia e meio ambiente, política de planejamento energético e modelagem da difusão de tecnologia, Vargas tem ocupado cargos e exercido funções de natureza científica, técnica e administrativa. Considerando que uma relação completa de suas atividades seria demasiado longa, citamos apenas as que nos parecem mais ilustrativas para a interdisciplinaridade das ciências: diretor do Instituto para Pesquisas Radioativas (Belo Horizonte); governador-adjunto, pelo Brasil, da Junta de Governadores da Agência Internacional de Energia Atômica (Viena); cientista visitante e líder de pesquisa do Comissariado de Energia Atômica (França); vice-presidente da Academia Brasileira de Ciências durante 14 anos, tendo assumido a presidência em várias oportunidades; presidente da Academia do Terceiro Mundo (Trieste); membro de algumas academias de ciências no exterior; ministro de Estado das pastas de Minas e Energia e da Ciência e Tecnologia. Vargas tem participado de vários organismos e comissões internacionais sendo que, desde março de 2000, exerce o cargo de embaixador da Delegação Permanente do Brasil junto à Organização Educacional, Científica e Cultural das Nações Unidas (Unesco).

Ieuda Ciornai (1922-86), químico industrial e engenheiro químico pela ENQ da UB, iniciou sua vida profissional na seção de FísicoQuímica do Laboratório da Produção Mineral, onde tomou contato com os métodos físico-químicos de análise. Em decorrência do uso cada vez mais freqüente de aparelhos eletrônicos, foi surgindo o interesse em se capacitar para prestar a assistência técnica necessária ao bom funcionamento dos instrumentos, todos importados, uma vez que não havia, na época, um serviço de manutenção satisfatório. Tornou-se, no Rio de Janeiro, o primeiro fabricante de instrumentos de laboratório com tecnologia própria. Primeiro tentou a fabricação com componentes facilmente encontráveis no mercado de rádio e televisão, tendo obtido patente relativa a um milivoltímetro eletrônico de alta impedância de entrada. Fundador e diretor técnico da firma Metronic Instrumentos Científicos, foi autor de todos os projetos de instrumentos fabricados pela empresa: medidor de $\mathrm{pH}$ (o primeiro inteiramente produzido no Brasil, incluindo a fabricação do vidro pH-sensível usado na montagem dos eletrodos), colorímetro fotoelétrico, condutivímetro etc. Lecionou na Escola de Química da UFRJ as disciplinas instrumentação industrial e teoria do controle de processos (Ciornai, 1982).

\section{A Faculdade Nacional de Filosofia e o Centro Brasileiro de Pesquisas Físicas}

Não se pode falar em interdisciplinaridade sem mencionar a FNFi da UB, fundada em 1939, ao mesmo tempo que era extinta a Universidade do Distrito Federal (UDF), cujos alunos e parte do corpo 
docente foram incorporados à recém-criada unidade. Com a estrutura de uma miniuniversidade e tendo seus vários departamentos funcionando num mesmo prédio (inicialmente no Colégio Estadual Amaro Cavalcanti, no Largo do Machado, e, em seguida, na antiga Casa D'Itália, hoje Consulado Geral da Itália, na Esplanada do Castelo), a FNFi atuou no Rio de Janeiro como elemento de integração entre discípulos e professores das diferentes áreas do conhecimento, da fundação ao fechamento (1968).

Ao ingressar no curso de química da FNFi, o aluno iniciava logo sua interação com o curso de física, pois durante dois anos as aulas da disciplina física geral e experimental eram ministradas em conjunto para esses dois cursos e também para o de matemática. O professor catedrático era Joaquim da Costa Ribeiro, que tinha como assistentes Elisa Frota Pessoa e, durante um certo período, Jayme Tiomno, que foi sucedido por Armando Dias Tavares.

A integração dos membros dos departamentos de Física e de Química era tão grande que levou Elza Vieira de Souza Teixeira (1992, p. 168 ), que terminou o curso de física em 1951, a assim se expressar em seu depoimento para o livro Faculdade Nacional de Filosofia: "Em relação à parte de pesquisa o que me lembro é de algo estranho. O Costa Ribeiro, em seus laboratórios... eu o vejo rodeado por uma equipe de estudantes de química. O grupo que trabalhava com o professor Costa Ribeiro, ao seu lado, era o grupo da química."

O próprio Costa Ribeiro (op. cit., p. 218), referindo-se ao fenômeno termodielétrico, relata estudos realizados por três alunos do curso de química da FNFi, os quais posteriormente também se diplomaram em física pela então Universidade do Estado da Guanabara (UEG): Edson Rodrigues, Sergio Mascarenhas e Yvonne Primerano (Mascarenhas, pelo casamento). Participava ainda desse grupo Mabel Medeiros Rodrigues. Essa reunião de estudantes de química em torno de seu professor de física gerou uma equipe de jovens idealistas que, anos mais tarde (no final da década de 1950 e no decorrer da de 1960), levaria a Escola de Engenharia de São Carlos (EESC) da Universidade de São Paulo (USP) a tornar-se palco de uma verdadeira revolução, com a implantação de atividades de pesquisa básica em física e em química. Daí resultou a criação, em 1968, do Instituto de Física e Química de São Carlos (IFQSC/ USP), que foi posteriormente desdobrado nas unidades atuais (IQSC e IFSC). As atividades de pesquisa, pós-graduação e graduação desses institutos, após registrarem notável crescimento qualitativo e quantitativo, resultaram em uma intensa produção científica.

Sergio Mascarenhas Oliveira, especialista em física do estado sólido, trabalhou também em termodinâmica dos processos irreversíveis, condutividade eletrotérmica, aplicações de eletretos à biofísica, além de outros assuntos em física e biofísica. Yvonne Mascarenhas, doutora em física pela EESC, especialista em cristalografia estrutural por difração de raios X, entrou em 2000 para a Academia Brasileira de Ciências. 
Edson Rodrigues, que atuou na EESC na cadeira de mecânica geral, com a constituição do IFQSC, ficou no Departamento de Química e Física Molecular, onde batalhou ativamente no desenvolvimento do programa de pós-graduação em físico-química. Mabel Rodrigues, ao ingressar nos quadros da Escola de Engenharia, optou pela cadeira de química geral e geologia. ${ }^{3}$ Milton Ferreira de Souza juntou-se mais tarde ao grupo de São Carlos, onde fez doutorado em física. Desempenhou também funções docentes na EESC, passando por todas as etapas da carreira do magistério, desde auxiliar de ensino até professor titular, chefe de departamento e diretor do Instituto de Física. Sua área de interesse é a física da matéria condensada.

É importante salientar que os comentários de Elza de Souza Teixeira referem-se ao período após a criação do CBPF, quando a pesquisa no campo da física começava a receber incentivos, e os jovens, motivados pelo novo panorama científico, ansiavam por se integrar a ele. Dispondo de uma equipe de pesquisadores dotada de grande preocupação com a formação de pessoal, além de possuir laboratórios bem equipados o que não se verificava na FNFi -, o CBPF exercia forte atração sobre os estudantes dos cursos de física e química. Além disso, ele recebeu, logo que foi fundado, um 'mandato universitário' que lhe permitia dar cursos básicos, os quais seriam reconhecidos pela UB. Mais tarde, houve o reconhecimento dos cursos de pós-graduação. Na qualidade de chefe do Departamento de Ensino do CBPF, Jayme Tiomno criou um Laboratório de Ensino no CBPF, onde os alunos de Elisa Frota Pessoa na FNFi tinham aulas práticas. Os dois primeiros chefes do laboratório foram Paulo Emidio Barbosa e Horacio Macedo (Tiomno, 1992, p. 269), ambos atuantes na área da físico-química.

Horacio Macedo (1925-99), químico industrial pela ENQ da UB, dedicou-se sobretudo ao ensino da físico-química, tendo lecionado na UFRJ, integrando o grupo do professor Zamith, e também na Universidade Federal Rural do Rio de Janeiro (UFRRJ). Seu primeiro emprego, porém, foi no Instituto de Manguinhos, atual Fundação Oswaldo Cruz (Fiocruz). Trabalhou ainda no CBPF e lecionou termodinâmica para químicos da Petrobras. Teve participação ativa na vida universitária da UFRJ, onde foi o primeiro reitor eleito. Entre suas obras mais conhecidas, destaca-se um dicionário de física.

Paulo Emidio Barbosa (1919-97), químico industrial pela Escola Nacional de Química, especializou-se em espectroscopia no Massachusetts Institute of Technology (MIT). No Laboratório da Produção Mineral (Rio de Janeiro), foi laboratorista, tecnologista e chefe da seção de físico-química, onde teve oportunidade de desenvolver estudos sobre espectrografia. Destacou-se por suas atividades acadêmicas na UFRJ, onde exerceu os cargos de diretor da Escola de Química, subreitor de Ensino de Graduação e Corpo Discente e decano do Centro de Ciências Matemáticas e da Natureza. Professor catedrático de física da Escola de Química, foi transferido, em decorrência da reforma 
universitária, para o Instituto de Física (IF). Após a aposentadoria, retornou à área da físico-química, ministrando, no curso de pósgraduação do Instituto de Macromoléculas, a disciplina físico-química de polímeros. Foi professor associado do CBPF e lecionou termodinâmica em cursos realizados em convênio entre a UB e a Petrobras (1952-70). Era membro associado da Academia Brasileira de Ciências.

$\mathrm{Na}$ FNFi, os estudantes e docentes de físico-química assistiam rotineiramente às palestras e seminários conduzidos por físicos, entre os quais podem ser citados José Leite Lopes, Cesar Lattes, Costa Ribeiro, Armando Dias Tavares, entre outros. Também era comum a participação em cursos extracurriculares, como, por exemplo, os de teoria quântica da valência e mecânica quântica para químicos, ministrados, respectivamente, por Ricardo Ferreira, no CBPF (1960-61), e Leite Lopes, na ENQ. Note-se que Ricardo Ferreira e Leite Lopes, físicos internacionalmente conhecidos, também tiveram sua primeira formação profissional em cursos de química. ${ }^{4}$

A físico-química era ensinada a partir do $2^{\circ}$ ano do curso de química, pelo grupo de Christovão Cardoso, catedrático da cadeira de físicoquímica e química superior. Alguns temas eram eventualmente desenvolvidos por professores convidados, destacando-se espectrografia, por Paulo Emidio Barbosa, e coloidoquímica, por Hans Zocher. Havia também, nos laboratórios da Físico-Química, aulas experimentais para alunos de outras unidades da universidade, como o Instituto de Eletrotécnica da Escola de Engenharia.

João Christovão Cardoso (1903-80), diplomado em medicina e desejando aperfeiçoar seus conhecimentos de física, cursou até a terceira série de engenharia, pois na época não existiam cursos específicos de física. Além dos conhecimentos de química e de física e de uma cultura humanística, Cardoso possuía larga experiência na indústria químicofarmacêutica, inclusive na área de produção. Foi também químicotecnologista do Instituto Médico-Legal. Era dotado de rara habilidade em improvisar aparelhos de física. Nos anos de 1945 e 1946, instalando as primeiras termas radioativas da América do Sul, no Hotel Quitandinha, em Petrópolis (RJ), teve oportunidade de fazer uso do eletrômetro bifilar de Wullf para medição de níveis de radiação. Suas atividades no magistério podem servir como mais um exemplo da interdisciplinaridade de física e físico-química. Além de ensinar físico-química (inicialmente na ENQ e depois na FNFi), era livre-docente de física biológica na Faculdade Nacional de Medicina, na UB, e professor de física em colégios de nível médio.

Embora não nos tenha legado uma extensa produção científica, Cardoso teve importante atuação nos meios universitário e científico, tendo sido membro da Academia Brasileira de Ciências, vice-presidente e, posteriormente, presidente do CNPq. Como representante da FNFi no Conselho Universitário, foi autor da proposta, aprovada em 1959, 
de criação do Instituto de Química (IQ) da UFRJ como centro de pós-graduação, com o objetivo de estimular a pesquisa e a formação de professores de nível superior. A Coordenação dos Cursos de Pós-Graduação em Engenharia (Coppe) - hoje Instituto Alberto Luiz Coimbra de Pós-Graduação em Engenharia — teve origem na Divisão de Engenharia Química do IQ, então dirigida por Alberto Luiz Coimbra. O Laboratório de Amido do INT, organizado por Ernesto Tolmasquim e Feiga R. Tiomno Rosenthal, teve origem no laboratório de físico-química da FNFi, onde, por iniciativa de Christovão Cardoso, foram realizados seminários e estágios sobre físico-química de macromoléculas naturais (1959-60). ${ }^{5}$

Bartyra de Castro Arezzo e Silvia Tiomno (posteriormente Tolmasquim), ambas bacharéis e licenciadas em química pela FNFi, faziam parte da equipe docente da cadeira de físico-química e química superior desta faculdade. Iniciaram suas atividades profissionais, ainda estudantes, como 'auxiliares de ensino sem remuneração', colaborando em aulas experimentais.

Christovão Cardoso, sensível aos progressos da tecnologia nuclear, incluiu em sua cátedra, na década de 1960, a disciplina opcional química nuclear e radioquímica, cuja ementa foi revista e atualizada no início dos anos 1970 e adotada pela Coppe, no curso de pós-graduação em engenharia nuclear, na opção química nuclear e radioquímica. Esses cursos foram ministrados por Bartyra Arezzo, que, em 1966, se afastou da FNFi e passou a integrar o quadro de pesquisadores da CNEN, onde desenvolveu trabalhos nos campos dos efeitos químicos das transformações nucleares e métodos físico-químicos de separação de radioelementos, como a extração por solventes e troca iônica.

Silvia Tiomno Tolmasquim continuava trabalhando com Cardoso na FNFi. Como os laboratórios não ofereciam condições para pesquisa, juntou-se informalmente ao grupo do Laboratório de Amido do INT, onde realizou estudos sobre propriedades físico-químicas de amidos e teve a oportunidade de elaborar a tese de livre-docência. No período de 1973 a 1983, afastou-se oficialmente da UFRJ, por requisição do INT, primeiro para coordenar os cursos de Especialização, Aperfeiçoamento e Extensão Universitária e, em seguida, para dirigir a Divisão de Ensino e Documentação. Retornou então à UFRJ onde permaneceu até a aposentadoria pelo IQ.

Com a reforma universitária, o IQ da UFRJ — que fora criado visando exclusivamente à pós-graduação — recebeu o encargo de ministrar também o ensino básico de química para toda a universidade. Extinta a FNFi (1968), o Departamento de Química (incluindo o grupo da físico-química) foi transferido para o IQ, que passou a funcionar na Ilha do Fundão. A partir daí, diminuiu o intercâmbio com o pessoal da área de física. Christovão Cardoso foi nomeado diretor pro tempore do IQ, função que exerceu junto com a chefia do Departamento de FísicoQuímica até a aposentadoria compulsória, em 1969, em decorrência do Ato Institucional nº 5 (AI-5). 
No ano seguinte, os docentes de físico-química da ENQ, inclusive Horacio Macedo, também foram transferidos para o IQ, tendo o professor catedrático Augusto Zamith sucedido Christovão Cardoso na chefia do Departamento de Físico-Química.

\section{Conclusão}

O presente trabalho é formado basicamente pelo depoimento das autoras sobre fatos vivenciados e observações feitas no decorrer de cerca de quatro décadas, primeiro na FNFi e depois no IQ/UFRJ, seja integrando o corpo discente da primeira ou o docente dessas duas unidades universitárias. Mas não foram desprezados os registros feitos por físicos, físico-químicos e historiadores da ciência na literatura especializada, ou em documentos pessoais.

Examinando as áreas de atuação de certos pesquisadores e professores, verifica-se uma verdadeira interpenetração e entrelaçamento de diferentes campos do saber, não havendo razão essencial para incluí-los entre físicos ou entre físico-químicos. Caberia a pergunta: Hervasio de Carvalho e Jacques Danon eram físicos ou físico-químicos?

Quanto ao inter-relacionamento dos jovens estudantes e pesquisadores que se dedicam a esses dois ramos da ciência, a situação atual parece-nos um tanto diferente daquela aqui exposta, com relação à extinta FNFi.

O antigo regime seriado permitia, mais do que o atual, um maior contato entre alunos de uma dada turma, bem como entre professores e seus discípulos. Embora o sistema de créditos tenha outros méritos, ao promover o desaparecimento da 'turma', ele determinou também a renovação dos colegas a cada disciplina cursada. Outro fator que contribuiu para desfavorecer a integração dos grupos com diferentes interesses científicos foi o crescimento quantitativo do corpo discente da universidade.

Há que se considerar ainda que atualmente o intercâmbio cultural das várias unidades universitárias da UFRJ é dificultado pela distância entre elas, pela estrutura arquitetônica da cidade universitária. Por outro lado, a grande influência exercida pelo CBPF — que, pode-se dizer, 'nasceu' dentro da FNFi — sobre o Departamento de Química desta faculdade já não se faz sentir com a mesma intensidade sobre o atual IQ, pela distância geográfica entre as duas unidades.

Ressaltamos porém que, embora a FNFi tenha desempenhado historicamente no Rio de Janeiro importante papel na integração dos diversos setores do conhecimento, sua extinção não significa, de forma alguma, o término da interdisciplinaridade de física e físico-química. Ao contrário, essas ciências vêm se desenvolvendo gradativamente na 
cidade e, à medida que se expandem e diversificam seus domínios, menos definidas ficam as fronteiras que as separam.

\section{Trabalhos publicados}

Apresentamos a seguir a seleção de alguns trabalhos da maioria das pessoas mencionados no artigo, pela ordem em que aparecem no texto. ${ }^{6}$ Para evitar que jovens leitores subestimem o peso da contribuição das primeiras gerações de físico-químicos, julgamos oportuno lembrar que a pesquisa sistemática no campo da físico-química, no Rio de Janeiro - e certamente no resto do país — só se desenvolveu após a efetiva implantação dos cursos de pós-graduação, por volta de 1970. Até então, grande parte dos trabalhos publicados era de natureza didática ou de divulgação científica. Como não existiam os cursos de mestrado e de doutorado, ${ }^{7} \mathrm{O}$ diploma de doutor era obtido por concurso de títulos e provas com defesa de tese, elaborada 'por conta própria', sem que existisse a figura do orientador. Geralmente a capacitação dos cientistas exigia, além de méritos pessoais, uma iniciação no campo da pesquisa no exterior, onde as condições do trabalho científico eram mais favoráveis que as nossas. Formaram-se assim competentes pesquisadores, com intensa produção científica, que, retornando ao Brasil, foram capazes de participar ativamente do desenvolvimento das novas gerações de cientistas.

José Carneiro Felippe

- 'Dosage de l'urée par la méthode comparative'. C. R. Soc. Biol., t. 96, $\mathrm{n}^{\circ} 37$, p. 1.948. 1926

- 'Radioactivité et action ologodynamique des minéraux'. C. R. Soc. Biol., t. 95, no 37, p. 1.948. 1926 (com Thales Martins)

- 'Inactivation et activation oligodynamique de l'argent'. C. R. Soc. Biol., t. 97, no 31, p. 1.364. 1927 (com Thales Martins)

- 'Análise pelo método de interpolação, das medidas de radioatividade'. $3^{\circ}$ Congresso Sul-Americano de Química, Rio de Janeiro, nº 2, p. 170. 1937

\section{João Christovão Cardoso}

Embora os trabalhos publicados não constem do currículo disponível na Academia Brasileira de Ciências, a biblioteca dessa entidade dispõe da separata da Revista de Química Pura e Aplicada (IV série, III ano, vol. XXXV, pp. 51-63), intitulada 'Estudos sobre a condução elétrica em hidrocarbonetos líquidos', de sua autoria (Porto, Imprensa Portuguesa, 1952). No Departamento de Informação e Documentação/Serviço de Arquivo do Mast-CNPq, encontra-se a tese apresentada ao concurso da cátedra de física do Colégio Pedro II: 'Em torno da lei de distribuição de Nernst' (Rio de Janeiro, 1963, 13p.).

Augusto Araujo Lopes Zamith 
- 'Interpretation statistique de l'isotherme de Freundlich dans le domaine de très faibles concentrations'. J. Chim. Phys., vol. 51, pp. 260-2. 1954 (com J. Danon)

- 'Solvent extraction of polonium from nitric acid solutions'. Nature, vol. 177, pp. 746-7. 1956 (com J. Danon)

- 'Ion-exchange and solvent-extraction studies with polonium'. J. Phys. Chem., vol. 61, pp. 431-4. 1957 (com J. Danon)

\section{Alberto Betim Paes Leme}

- 'A espectrografia e o novo metal da Casa da Moeda'. Rev. Chim. Phys., ano I, no 7. 1916.

- Análise espectral aplicada à mineralogia. Rio de Janeiro, Papelaria Macedo, 138 p. ilus. 1918

- 'Le Germanium dans les météorites; analyse spectrale quantitative du germanium dans les météorites Santa Luzia (État de Goyaz) et Bendegó (État de Bahia) Brésil'. Boletim do Museu Nacional, vol. X, pp. 113-4. 1934

- 'Análise espectral quantitativa do germânio nos holosidentos brasileiros Bendengó e Santa Luzia'. Anais da Academia Brasileira de Ciências, VII:2, pp. 177-9. 1935

$\mathrm{Na}$ biblioteca da Academia Brasileira de Ciências, encontra-se a publicação Aplicação do estudo fisicoquímico das ligas ferro-carbono a um aço obtido por um processo novo do professor Ennes de Souza (Rio de Janeiro, Papelaria Macedo, 1918, 51p.).

\section{Ernesto Lopes da Fonseca Costa}

- 'Relatório dos trabalhos feitos durante o ano de 1922 na Estação Experimental de Combustíveis e Minérios'. Rio de Janeiro, Imprensa Nacional, 30p. 1926

- 'Novo método de determinação das velocidades de reação em função da temperatura'. Anais da Academia Brasileira de Ciências, 7:1, pp. 57-62. mar. 1935

- 'Notas em torno do problema siderúrgico nacional'. Rio de Janeiro, INT, 16p. 1935

- Congresso Sul-Americano de Química, 3. Rio de Janeiro/São Paulo. 1937. Atas e trabalhos. Nota sobre a cinemática do sistema Fe-O-C. Rio de Janeiro, Serviço de Informação Agrícola, vol. 2, pp. 98-105. 1941

- 'Indústria nacional de enxadas'. R. Quim. Ind., 19:221, pp. 24-5. set. 1950

- Oálcool como combustível industrial. Rio de Janeiro, INT, 23p. 1927

- Possibilidades econômicas do carvão de Santa Catarina. Rio de Janeiro, INT, 67p. 1928

- O carburante nacional. Rio de Janeiro, INT, 14p. 1932 
- 'Localização de uma fábrica de tubos de aço sem costura'. B. Int., 4:10, pp. 3-19. set. 1953

- Estudo do manganês e sua metalurgia, sob o ponto de vista brasileiro. Tese, Rio de Janeiro, Pap. Americana, 65p. 1929

- 'Instituto Nacional de Tecnologia e seus fins'. Technologia, 1:1, pp. 16-29. set. 1934

- 'Termo Balança'. Technologia, 1:4, pp. 5-9. jan.fev. 1935

O trabalho 'O álcool como combustível industrial' é uma conferência proferida em 1925, na então Escola Politécnica do Rio de Janeiro. Em 1979, pela atualidade do assunto face à implantação do Próalcool, o Informativo do INT (vol. XII, no 22, pp. 3-23), periódico editado pelo instituto, republicou o trabalho ilustrado com a fotografia do automóvel Ford que em 1925, após ter sido especialmente preparado na Estação Experimental de Combustíveis e Minérios (hoje INT), fez os percursos Rio - São Paulo, Rio - Barra do Piraí e Rio - Petrópolis alimentado com álcool $70^{\circ} \mathrm{GL}$.

\section{Alvaro Alberto da Mota e Silva}

- 'Considerações sobre a estabilidade química da nitrocelulose, da nitroglicerina e das pólvoras sem fumaça'. $R$. de Ciências, vol. 6, pp. 11-16. jan.-dez. 1922

- 'A propósito do ensaio de Angeli (pesquisa da acidez nas pólvoras sem fumaça)'. R. de Ciências, vol. 6, pp. 16-20. jan.-dez. 1922

- 'Sobre as dimensões dos cristais formados em meio viscoso'. Anais da Academia Brasileira de Ciências, 1:1, pp. 1-4. mar. 1929

- 'Sobre a estabilidade dos cristais e seus calores de formação e dissolução'. Anais da Academia Brasileira de Ciências, 1:1, pp. 179. mar. 1929

- 'Sobre uma expressão analítica da regra de Van't Hoff'. Anais da Academia Brasileira de Ciências, 1:3, pp. 138-41. set. 1929

- 'Sobre a reconcentração de banhos sulfonítricos'. Anais da Academia Brasileira de Ciências, 2: 3, pp. 113-29. set. 1930

- 'Sobre o resíduo de carbono em certas explosões'. Anais da Academia Brasileira de Ciências, 2:4, pp. 187-8. dez. 1930

- Pólvoras sem dissolvente volátil. Rio de Janeiro, Ministério da Guerra, 2 vols. 1939

- À margem da ciência. Rio de Janeiro, Imprensa Naval, 4 vols. 196072

Entre 1932 e 1946, Alvaro Alberto publicou nos Anais da Academia Brasileira de Ciências diversos estudos enfocando temas como reações entre sólidos, vaselinas nas pólvoras propulsivas, poder erosivo das pólvoras, erosão das armas de fogo, além de outros (inclusive um sobre 'Aristóteles e a queda dos corpos').

Hans Ernst Werner Zocher 
- Ueber zinnsaure und bleisaure Salze. Dissertação, Leipzig, Leopoldo Voss, 71p. 1920. Zs. anorg. Chem., vol. 112, p. 1. 1920

- 'Ueber Sole mit nichtkugeligen Teilchen'. Zs. Physik. Chem., vol. 98, p. 293. 1921

- 'Ueber die Beziehung zwischen Chemi — und Photoluminiszens bei ungesättigen Siliziumverbindungen'. Zs. Phys., 9:5, pp. 267-84. 1922 (com H. Kautsky)

- 'Ueber Luminiszenz bei chemischen Reaktionen'. Naturwissench, $\mathrm{n}^{\circ}$ 11, pp. 194-9. 1923 (com H. Kautsky)

- 'Über Gele des Chinis und seiner Derivate'. Biochem. Zeitschrift, 137:4-6, pp. 413-28. 1923. (com P. Rona e E. Schmerl)

- 'Kolloidchemische Beobachtungen an Salvarsan und Neosalvarsan'. Biochem. Zs., 138:4/6, pp. 307-17. 1923 (com H. Freundlich e R. Stern)

- 'Ueber das Wesen der Chemiluminiszenz'. Zs. für Elektrochemie, 29:13-14, pp. 141-4. 1923 (com H. Kautsky)

- 'Quantitative Untersuchungen am Vanadinpentoxyd, I. Die Stoemungsanisotropie'. Zs. physik. Chem., 114:3-4, pp. 161-89. 1924 (com H. Freundlich e F. Stapelfeld)

- 'Quantitative Untersuchungen am Vanadinpentoxyd, II. Das Wirbelkreuz'. Zs. physik. Chem, 114:3-4, pp. 190-207. 1924 (com H. Freundlich e F. Stapelfeld)

- 'Ueber die Entstehung des Glanzeindruckes'. Zs. Physik, 33:1-2, pp. 12-27. 1925 (com F. Reinicke)

- 'Ueber freiwillige Strukturbildung in Solen'. Zs. anorgan. allg. Chem., 147:1-3, pp. 91-110. 1925

- 'Ueber die Erzeugung optischer Aktivität an Silber durch zircular polarisiertes Licht'. Sitzungsber. preuss. Akad. Wissenschaften, vol. 23, pp. 426-31. 1925 (com K. Coper)

- 'Optischen Methoden zur Untersuchung der Anisotropie in Kolloiden'. Kolloid-Zs., 37:6, pp. 336-51, 1925

- 'Ueber die Elastizitaet und die Stroemungsdoppelbrechung in Solen mit nichtkugelingen Teilchen: 1'. Kolloid-Zs, 38:1, pp. 43-54. 1926 (com H. Freundlich e H. Neukircher)

- 'Anisotropes Kupfer, Silber und Gold. Zs. Physik. Chem., 124:1-2, pp. 83-96. 1926 (com S. Berkmann e J. Böhm)

- 'Ueber das magnetische Verhalten verschiedener Stoffe'. Zs. physik. Chem., 124:5-6, pp. 318-26. 1927 (com S. Berkmann)

- 'Ueber frewillige Strukturbildung im Vanadinpentoxidsol'. KolloidZs., 41:3, pp. 220-1. 1927 (com K. Jacobsohn)

- 'Zur Kenntniss der physikalisch-chemischem Eingeschaften der Merkurisulfosalizylate, 1 Teil'. Kolloid-Zs., 42:4, pp. 309-28. 1927 (com S. Berkmann)

- 'Über die Passivität von Eisenspiegeln, I. Chemischer Teil'. Zs. physik. Chem., 128:5-6, pp. 321-44. 1927 (com H. Freundlich e G. Patschekke) 
- 'Über die Passivität von Eisenspiegeln, II. Optischer Teil'. Zs. physik. Chem., 130: 5-6, pp. 289-307. 1927 (com H. Freundlich e G. Patschekke)

- 'Über die Einwirkung elektrischer, magnetischer und mechanischer Kräfte auf Mesophasen'. Physikalische Zs., vol. 28, pp. 790-6. 1927

- 'Über die Erzeugung von Anistropie von Oberflächen'. Zs. physik. Chem., 132:3-4, pp. 295-312. 1928 (com K. Coper)

- 'Über die Erzeugung optischer Aktivität durch zirkulares Licht'. Zs. physik. Chem., 132:3-4, pp. 313-9. 1928 (com K. Coper)

- 'Ultramikroskopie'. Methodik der wissenschftlichen Biologie, vol. 1, pp. 417-58. 1928.

- 'Über Strukturbildung in kolloidem Lösungen'. Collegium, vol. 696, p. 203.1928

- 'Ueber Sol-Gel Systeme mit anisotropen Teilchen, I. Dibenzoylzystin'. Kolloid-Zs., 46:1, pp. 27-33. 1928 (com H. W. Albu)

- 'Ueber Sol-Gel Systeme mit anisotropen Teilchen, II. Bariummalonat'. Kolloid-Zs., 46:1, pp. 33-6. 1928 (com H. W. Albu)

- 'Einfluss photographischer Reaktionen auf den Weigert-Effekt in Photochlorid'. Zs. physik. Chem., vol. 139, pp. 218-63. 1928 (com K. Coper)

- 'Über den Einfluss photographischer Reaktionen auf den WeigertEffekt in Photochlorid'. Zeitschrift fürphysikalische Chemie, 141:3, pp. 217-8 (separata). 1929 (com K. Coper)

- 'Kurze Mitteilung über die Doppelbrechung von Bentonit'. KolloidZs., 47:3, p. 223. 1929 (com R. Bradfield)

- 'Über Taktosole'. Kolloidchem. Beih., 28: 6, pp. 167-205. 1929 (com K. Jacobsohn)

- 'Über die optische Anisotropie des gedehnten Kautschuks'. Kautschuk, no 8, p. 173.1929

- 'Dunkelfeldmikroskopie monomolekularer Filme'. Vorlaeufige Mitteilung, Naturwissenschaften, 17:34, pp. 672-3. 1929 (com F. Stiebel)

- 'Schillerschichten als Reaktionsprodukte der langsamen EisenchloridHydrolyse'. Zs. anorg. allg. Chem., vol. 186, pp. 57-96. 1930 (com W. Heller)

- 'Dunkelfeldmikroskopie dünnster Filme auf Flüssigkeitsoberflächen'. Zeitschrift für physikalische Chemie, 147:6, pp. 401-35 (separata). 1930 (com F. Stiebel)

- 'Über die Nachweisbarkeit der Strömungs-Doppelbrechung in sehr kleinen Konzentrationen'. Zs. wissenschaftl. Photographie, 29:1-12, pp. 102-9. 1931 (com H. Freundlich e I. V. Tamchyna)

- 'Optik der Mesophasen'. Zs. Kristallographie, 79:1-4, p.122-33. 1931

- 'Über die Struktur der aus dem Molekuelstrahl entstandenen Schichten'. Zs. Elektrochem., 37:8-9, pp. 571-7. 1931 (com K. Coper e L. Frommer) 
- 'Effect of a magnetic field on the nematic state'. Transact. da Faraday Soc., vol. 29, pp. 945-57. Sept. 1933

- 'Über die transversale magneto-optische Anisotropie einiger kolloidalen Lösungen, I'. Zs. physik. Chem. A., 164:1-2, pp. 55-79. 1933 (com W. Heller)

- 'Untersuchungen über Feinstruktur das Holzes, I. Die Volumenquellung von Fichtenholz und Lignin in verschiedenen Medien'. Kolloidchem. Beih., 40:7-12, pp. 413-48. 1934 (com H. J. Saechtling)

- 'IV. Optik disperser Systeme'. Em Kolloidchemichs Taschenbuch. Leipzig, Akademische Verlag., pp. 77-100. 1943

- 'Untersuchungen über Feinstruktur des Holzes, II. Die dampfdruckerniedrigung verschiedener Fluessiggkeiten bei ihrer Augnahme durch Holz und Lignin'. Kolloidchem. Zs., 72:3, pp. 33645. 1935 (com H. J. Saechtling)

- 'Freiwillige Ordung von Molekülen oder Rolloidtreichen zu Faserstrukturen'. Prag: Deutschen agrar. Druckerei, 10p. 1936

- 'Über die Anwentbarkeit der Theorie der thermischen Schwankungen auf die Gebiet der Mesophasen'. Kolloid-Zs., 75:2, pp. 161-3. 1936

- 'Beitrag zur Theorie der Schillerschichten'. Zs. physik. Chem. A., vol. 181, pp. 301-14. 1938 (com P. Bergmann e P. Loew Beer)

- 'Über die Kontinuumtheorie und die Schwarmtheorie der nematischen Phasen'. Ann. Phys., ano 5, 31:5, pp. 570-8. 1938

- 'Über die Struktur undeformieter und einfach deformieter Gebiete in nematischen Schichten grosserer Dicke'. Zs. Physik, 110:9-10, pp. 529-48. 1938 (com G. Ungar)

- 'On the polarization of the fluorescence of dyestuffs dissolved in mesophases'. Trans. da Faraday Soc., 35:213, pp. 34-7. Jan. 1939

- 'Kritische Untersuchungen zur Analyse von Hyposulfit-Präparaten'. Zeitschrift für analytische Chemie, 117:11-12 (separata). 1939 (com H. J. Saechtling)

- 'Algumas experiências sobre relações entre a fluorescência e adsorção'. Anais da Associação Química do Brasil, 19 p. (separata). 1949. Boletim do L.P.M., vol. 35. 1949 (com C. Török)

- 'Zur Kenntnis der Fluoreszens von Salzen des 8-Oxychinolins'. Monatshefte für Chemie, 81:1-2, pp. 274-9 (separata). 1950 (com F. Feigl e C. Török)

- 'About space-time asymmetry in the realm of classical general and crystal Physics'. Proceedings da National Academy of Sciences, 39:7, pp. 681-6 (separata). July 1953 (com C. Török)

- 'Räumliche und zeitliche Asymmetriebetrachtungen in der Physik, insbesondere der Kristallphysik'. Zeitschrift für Physik, vol. 139, pp. 147-62 (separata). 1954 (com C. Török)

- 'Taktosole und Mesophasen'. Kolloid-Zeitschrift, 139:1-2, pp. 81-5 (separata). 1954 
- 'Optischen Asymmetrien'. Zeitschrift für Physik, vol. 142, pp. 60218 (separata). 1955 (com C. Török)

- 'Beziehungen zwischen Klassischen und Relativistischen Invarianzoperationen'. Notas de Física, Rio de Janeiro, 4:1, 13p. (separata). 1958 (com C. Török e G. Beck)

- 'Observations on the solidification of paraffin'. Acta Crystallographica, 12:2, pp. 122-5 (separata). Feb. 1959 (com R. D. Machado)

- 'Optik kolloider Systeme'. Kuhn, Kolloidchemisches Taschenbuch, pp. 176-221 (separata). 1960

- 'Optische Aktivität'. Kubn, Kolloidchemisches Taschenbuch, pp. 2216 (separata). 1960

- 'Neuere Beiträge zur Kenntnis der Taktosole. Teil I. Ein neues taktosol: Aluminiumhydroxyd'. Kolloid-Zeitschrift, 170:2, pp.140-4 (separata). 1960 (com C. Török)

- 'Neuere Beiträge zur Kenntnis der Taktosole.Teil II.: Weitere Fälle von Aluminiumhydroxyd-Taktosol und -Taktogel, sowie von komplizierteren Taktoiden'. Kolloid-Zeitschrift, 173:1, pp. 1-7 (separata). 1960 (com C. Török)

- 'Neuere Beiträge zur Kenntnis der Taktosole. Teil III. Aluminiumhydroxid-Taktosole mit Querstruktur'. Kolloid-Zeitschrift, Zeitschrift für Polymere, 180:1, pp. 41-51 (separata). 1962 (com C. Török)

- 'Emploi de la méthode de Foucault-Toepler avec le microscope de Kofler'. Revue d'Optique, 42:9, pp. 447-50. Sept. 1963 (com R. D. Machado)

- 'Crystals of higher order and their relation to other superphases'. Acta Crystallographica, 22:6, pp. 751-5 (separata). June 1967 (com C. Török)

- 'Some topics of liquid crystals yet to be discussed'. Molecular Crystals and Liquid Crystals, vol. 7, pp. 165-80 (separata). 1969

- 'Über unsymetrische Metallkolloide'. Metallwirtschaft, nํ 20, pp. 467-9. März 1929

\section{Hervasio Guimarães de Carvalho}

De 1937 a 1949, Hervasio de Carvalho manifestou interesse específico pela química e físico-química, abordando temas dessas áreas em seus trabalhos. Entre 1948 e 1952, desenvolveu estudos no campo da radioatividade, com ênfase no uso de emulsões fotográficas para identificar e determinar quantitativamente elementos radioativos. Finalmente direcionou-se totalmente para a física nuclear, onde se encontra a maior parte de sua produção científica, havendo porém trabalhos que se situam na interface física/físico-química, mesmo nesta última fase.

- 'Estudos conseqüentes da fórmula de Nernst'. Revista Brasileira de Quimica, no 20, p. 191. 1937 
- 'Análise qualitativa do nitrogênio nas substâncias orgânicas'. Revista Brasileira de Química, no 48, p. 256. 1938

- Espectrografia do efeito Smekal-Raman na química analítica orgânica. Tese. 1939

- 'Relação entre a viscosidade e a temperatura'. Anais da Associação Química do Brasil, 2:1, p. 21. 1943

- 'Contribuição ao estudo físico-químico da água'. Anais da Associação Química do Brasil, vol. 3, p. 154. 1944

- 'Pressão interna, pressão de vapor e tensão superficial dos líquidos'. Anais da Academia Brasileira de Ciências, t. XX, no 1.1948

- 'Contribuição à físico-química das soluções diluídas'. Anais da Academia Brasileira de Ciências, t. XX, no 1, p. 75. 1948

- 'Variação da viscosidade dos gases com a pressão'. Anais da Academia Brasileira de Ciências, t. XX, nº 1.1948

- 'Identificação e análise de pequena quantidade de urânio e tório empregando emulsões nucleares'. Anais da Associação Química do Brasil, vol. VIII, p. 67. 1948

- 'Identificação de minerais alfa radioativos pelo método das chapas fotográficas especiais'. Anais da Associação Química do Brasil, vol. VIII, p. 84.1948

- 'Efeito da temperatura na sensibilidade das chapas fotográficas para física nuclear'. Anais da Associação Química do Brasil, vol. VIII, p. 102. 1948

- 'Stopping power of nuclear emulsion for Ionizing particles'. Physical Review, no 76, p. 1.729. 1949

- 'Peso molecular dos líquidos na temperatura de ebulição'. Boletim do DNPM, no 35, p. 129. 1949

- 'Range of alpha particles in water and ice'. Physical Review, $\mathrm{n}^{\mathrm{0}} 78$, p. 330. 1950

- 'Stars and heavy primaries recorded during V-2 rocket flight'. Physical Review, no 78, p. 765.1950 (com Herman Yagoda e Nathan Kaplan)

- 'The range of alpha particles in water'. Physical Review, no ${ }^{\circ 8} .1952$ (com H. Yagoda)

- 'Dosagem de monazita pelo contador Geiger Müller'. Anais da Associação Brasileira de Química, vol. XII, p. 35.1952 (com Washington M. de Amorim)

- 'Gradation development for the nuclear emulsions'. Physical Review, no 91, p. 224. 1953 (com V. Davis e W. Tesh)

- 'Polarization by p-p collision at 310 MeV'. Physical Review, n 93, p. 1.431. 1954 (com J. Marshall e L. Marshall)

- 'Polarization by p-p collision at 439 MeV'. Physical Review, no 94, p. 1.796. 1954 (com E. Heiberg, J. Marshall e L. Marshall)

- 'Total cross sections of $208 \mathrm{MeV}$ and $315 \mathrm{MeV}$ protons for light elements'. Physical Review, no 96, p. 398. 1954

- 'Polarization of protons by scattering from beryllium'. Physical Review, $\mathrm{n}^{\mathrm{o}}$ 96, p. 1.081. 1954 (com J. Marshall e L. Marshall) 
- 'Some recent results concerning fission and fragmentation at high energy'. Nuclear Physics, no 53, p. 345. 1964 (com G. Cortini, R. Rinzivillo e S. Sassi)

- 'Photofission of uranium, thorium and bismuth at energies of 300 to 1000 MeV'. Nuclear Physics, no 73, p. 385.1965 (com F. Carbonaro, R. Rinzivillo, E. Sassi e C. P. Murtas)

- 'U-238 fission induced by low-energy gamma rays, cross-sections between 5 and 8 MeV'. Nuclear Physics, no 74, p. 377.1965 (com A. Manfredini, M. Muchinik, L. Fiore, C. Ramorino, J. Lang e R. Müller)

- 'Gamma-neutrons reactions in the energy range of 1.0-5.5 GeV'. Nuclear Physics, no 136, pp. 505-12. 1968 (com V. Di Napoli, D. Margadonna, F. Salvetti e K. Tesch)

- 'Angular distribution of U-238 photofission fragments for 12 different monoenergetic gamma-rays'. Nuclear Physics, no 123, pp. 664-72. 1969 (com A. Manfredini, L. Fiore, C. Ramorino e W. Wolfli)

- 'Cross section of Th-232 photofission for 12 different monoenergetic gamma rays'. Nuclear Physics, no 127, pp. 687-92. 1969 (com A. Manfredini, L. Fiore, C. Ramorino e W. Wolfli)

- Radioactive decay of radium and radon isotopes by C-14 emission'. Physical Review, no 34, p. 6. 1986 (com J. B. Martins e O. A. P. Tavares)

Podem ser mencionados ainda os diversos artigos publicados entre 1958 e 1978, em diferentes periódicos como Il Nuovo Cimento, Notas de Física, Gazetta Chimica Italiana, Journal of Inorganic Nuclear Chemistry, Anais da Academia Brasileira de Ciências e outros, versando sobretudo sobre emulsões nucleares, fotofissão, fissão nuclear induzida e reações fotonucleares.

\section{Sergio Pereira da Silva Porto}

- The infrared emission spectrum of molecular hydrogen. Dissertação, Baltimore, Johns Hopkins University. 1954

- 'Infrared spectrum of hydrogen and deuterium between one and two microns'. J. Opt. Spc. Am., no 45, p. 447. 1955 (com G. H. Dieke)

- The molecular hydrogen spectrum in the near infrared. Tese apresentada à Faculdade Nacional de Filosofia, Rio de Janeiro, Universidade do Brasil. 1955

- 'Fine structure of some infrared bands of $\mathrm{CH}_{2} \mathrm{~F}_{2}$ and $\mathrm{CH}_{2} \mathrm{ClF}$ '. $J$. Molecular Spectroscopy, no 3, p. 248. 1959

- 'The infrared spectrum of the diethylbenzenes'. Anais da Academia Brasileira de Ciências, no 29, p. 345. 1958

- 'Determination of interatomic distances for rotational spectrum'. Anais da Academia Brasileira de Ciências, no 29, p. 323.1958 (com W. A. Cunha) 
- 'The comet tail band system of $\mathrm{CO}^{+}$. Anais da Academia Brasileira de Ciências, no 30, p. 133. 1958

- 'Determination of the asymmetry constant of slightly asymmetrical top molecules'. Anais da Academia Brasileira de Ciências, nº 31, p. 31. 1959 (com N. Jannuzzi)

- 'A high resolution grating spectrometer'. Anais da Academia Brasileira de Ciências. 1960 (com A. Szulc, J. P. Rezende Neto e W. O. N. Guimarães)

- 'Table of energy levels of a slightly asymmetrical top rotor'. J. Molecular Spectroscopy, no 4, p. 449.1960 (com N. Jannuzzi)

- 'Ruby optical maser as a Raman source'. J. Opt. Soc. Am., no 52, p. 251. 1962 (com D. L. Wood)

- 'Optical maser characteristics of $\mathrm{BaF}_{2}$ : $\mathrm{U}^{+3}$ '. Proc. IRE, vol. 50, p. 1.542. 1962 (com A. Yariv)

- 'Molecular constants of the ground state of $\mathrm{H}_{2}$ derived from theoretical calculations'. J. Chem. Phys., no 36, p. 2.520. 1962 (com C. A. Lambert)

- 'Optical maser emission from trivalent praseodymium in calcium tungstate'. J. Appl. Phys., no 33, p. 2.519. 1962 (com A. Yariv e K. Nassau)

- New singlet transitions in the near infrared spectrum of molecular hydrogen'. J. Mol. Spectr., no 11, p. 378. 1963 (com N. Jannuzzi)

- 'Continuous helium-neon red laser as a Raman source'. J. Opt. Soc. Am., no 53, p. 1.446. 1963 (com H. Kogelnit)

- 'Low lying energy levels and comparison of laser action of $\mathrm{U}^{+3}$ in $\mathrm{CaF}_{2}$. Em Grivet e Bloembergen (orgs.), Quantum Electronics III. Columbia University Press, p. 717. 1964 (com A. Yariv).

- 'Nonradiative energy exchange and laser oscillation in $\mathrm{Yb}^{3+} \mathrm{Nd}^{3+}$ borate glass'. Appl. Phys. Lett., nº 4, p. 202. 1964 (com A. D. Pearson)

- 'Use of a gas laser in studies of the depolarization of the Rayleigh scattering from simple liquids'. J. Chem. Physics, nº 40, p. 3.741. 1964 (com R. C. C. Leite e R. S. Moore)

- 'The laser as a Raman source - a survey'. Ann. N. Y. Acad. Sciences, no 122, p. 643.1965

- 'Angular dependence of the Rayleigh scattering from low turbidity molecular liquids'. Phys. Rev. Lett., no 12, p. 7.1965 (com R. C. C. Leite, R. S. Moore e J. E. Ripper)

- 'A tandem spectrometer to detect laser excited Raman radiation'. Appl. Optics, no 4, p. 762.1965 (com D. Landon)

- 'Laser excited Raman spectra of deuterated $\mathrm{KH}_{2} \mathrm{PO}_{4}$ ' J. Phys. Chem. of Sol., no 26, p. 2.085. 1965 (com I. P. Kaminov e R. C. C. Leite)

- 'The Raman effect of ZnO'. Phys. Rev., no 142, p. 570.1966 (com T. C. Damen e B. Tell)

- 'Angular dependence and depolarization ratio of the Raman effect'. J. Opt. Soc., no 56, p. 1.585. 1966 
- 'Enhancement of Raman cross section in CdS due to resonant absorption'. Phys. Rev. Lett., no 17, p. 10.1966 (com R. C. C. Leite)

- 'High resolution Raman effect of gases with laser sources'. J. Opt. Soc. Am., no 57, p. 19. 1967 (com A. Weber, L. E. Cheesman e J. H. Barret)

- 'The thermal lens effect as a power-limiting device'. Appl. Phys. Lett., no 10, p. 100. 1967 (com R. C. C. Leite e T. C. Damen)

- 'The Raman effect of corundum'. J. Chem. Phys., no 47, p. 1.009. 1967 (com R. S. Krishnan)

- 'Longitudinal and transverse optical lattice vibrations in quartz'. Phys. Rev., no 161, p. 903.1967 (com J. F. Scott)

- 'Light scattering by spin waves'. J. Appl. Phys., no 39, p. 1.035. 1968 (com P. A. Fleury)

- 'Rayleigh scattering of linearly polarized light from optically active quartz'. Appl. Opt., no 7, p. 1.913. 1968 (com C. A. Arguello e D. L. Rousseau)

- 'Optical phonons of yttrium aluminum garnet'. Phys. Rev., no 173, p. 851. 1968 (com J. P. Hurrell, I. F. Chang, S. S. Mitra e R. P. Bauman)

- 'Selection rules for the electronic Raman effect'. J. Mol. Spectr., no 32, p. 458. 1969 (com A. Kiel)

- 'Optical phonons in sodium chlorate'. Phys. Rev., no 188, p. 1.328. 1969 (com C. H. Hartwig e D. L. Rousseau)

- 'Laser Raman spectroscopy'. Ind. Res., nº 11, p. 67.1969

- 'Polywater: polymer or artifact?'. Science, no 167, p. 1.715. 1970 (com D. L. Rousseau)

- 'Photoluminescence studies in superconducting $\mathrm{Nb}_{3} \mathrm{Sn}$ '. Sol. State Com., no 8, p. 2.113. 1970 (com L. M. Fraas e F. Williams)

- 'An interferometric rejection filter for Raman spectroscopy'. Appl. Opt., no 10, p. 531.1971 (com W. Proffitt, L. M. Fraas e P. Cervenka)

- 'Some effects of impurities on near resonant Raman scattering from semiconductors'. Em M. Balkanski (org.), Light scattering in solids. 1971 (com P. F. Williams)

- 'Photoluminescence as a probe of band structure in metals'. Em M. Balkanski (org.), Light scattering in solids, op. cit., 1971 (com L. M. Fraas)

- 'Physics of lasers'. Em M. Feld e A. Javan (orgs.), Proc. Esfahan Symposium on Fundamental and Applied Laser Physics. 1971 (com W. G. Harter)

\section{Jacques Abulafia Danon}

- 'Rôle de la polarization prealable dans l'electrolyse des solutions extremement diluées'. J. Chim. Phys., vol. 47, p. 62.1950 (com M. Haissinsky)

- 'Dépôts electrolytiques de protoactinium sur divers métaux'. C. $R$. Acad. Sci., vol. 234, pp. 1.361-3. 1952 (com C. Ferradini) 
- 'Dépôts cathodiques en couches monoatomiques de bismuth sur le platine et l'or'. J. Chim. Phys., vol. 49, pp. 363-5. 1952 (com A. Coche e M. El Guebely)

- 'Remarques sur les methodes statistiques de derivation de la function de distribution de l'energie d'adsorption sur une surface hétérogène'. J. Chim. Phys., vol. 52, pp. 392-5. 1955

- 'Sobre a carga do íon complexo de proto-actínio em meio fluorídrico'. Anais da Academia Brasileira de Ciências, vol. 27, p. 91. 1956

- 'Absorption of thorium by anion-exchange resins from nitric acid media'. J. Am. Chem. Soc., vol. 78, pp. 5.953-4. 1956

- 'Échanges isotopiques et évolution des surfaces métalliques dans des solutions électrolytiques'. J. Chem. Phys., vol. 54, pp. 527-32. 1957 (com U. Camerini e M. Malagolowkin)

- 'Anion-exchange studies with actinium and lanthanides in nitrate solutions'. J. Inorg. Nucl. Chem., vol. 7, pp. 422-4. 1958

- 'Paper chromatography of inorganic ions in nitrate solutions: I. scandium, yttrium, actinium and the lanthanides'. J. Chromatog., vol. 3, pp. 193-4. 1960 (com M. C. Levi)

- 'Determination of the stability constants of thorium nitrate complexes with anion-exchange resins'. J. Inorg, Nucl. Chem., vol. 13, pp. 1128. 1960

- 'Espectro infravermelho de nitroprussiato de sódio irradiado por elétrons'. Anais da Academia Brasileira de Ciências, vol. 38, p. 577. 1960 (com L. F. Medina)

- 'Formação de depósitos eletrolíticos de nióbio, tântalo e zircônio em meio de fluoreto sobre diversos metais', Anais da Academia Brasileira de Ciências, vol. 33, p. 36. 1961 (com M. C. Levi)

- Paper chromatography of inorganic ions in nitrate solutions: separation of Ca-Ba-Sr and Ra'. J.Chromatog., vol. 6, p. 269. 1961 (com M. C. Levi)

- 'Estabilidade dos complexos de lantanídeos com ligante nitrato'. Anais da Academia Brasileira de Ciências, vol. 34, p. xxi-R. 1962 (com I. G. Almeida)

- 'Measurement of nuclear quadrupole interactions in iron complexes using the Mössbauer effect'. J. Phys. Chem. Solids, vol. 23, pp. 1.7835. 1962 (com N. L. Costa e R. M. Xavier)

- 'Nuclear isomer shift and covalency in iron complexes'. J. Chem Phys, vol. 39, pp. 236-7. 1963

- 'Nuclear isomer shift and nephelauxetic effect in iron complexes'. Rev. Mod. Phys., vol. 36, p. 459. 1964

- 'Mössbauer effect with trivalent iron halides in different molecular symmetries'. Rev. Mod. Phys., vol. 36, p. 460. 1964 
- 'Electron spin resonance of trapped electrons in irradiated sodium nitroprusside'. J. Chem. Phys., vol. 41, pp. 3.651-2. 1964 (com R. P. A. Muniz e M. Panepucci)

- 'Electron spin resonance of the $\mathrm{N}_{2} \mathrm{O}_{2}{ }^{+}$radical'. Mol. Phys., vol. 9, pp. 599-600. 1965 (com R. P. A. Muniz)

- 'Espécies paramagnéticas formadas pela irradiação do nitroprussiato de sódio'. Anais da Academia Brasileira de Ciências, vol. 38, p. 584. 1966 (com R. P. A. Muniz e A. O. Caride)

- 'Mössbauer hyperfine interactions in sodium nitroprusside single crystals'. J. Chem. Phys., vol. 47, pp. 382-7. 1967 (com L. Iannarella)

- 'Estudio por EPR de monocristales de ferrocianuro de potassio irradiados'. V Reunión de la Associación Fisica Argentina, Buenos Aires. 1968 (com A. O. Caride e S. I. Zanette)

- 'Deslocamento isomérico em ligas metálicas'. II Simpósio de Física Teórica da PUC, Rio de Janeiro, vol. 7, p. 157. 1969 (com S. A. D. Silva e A. A. Gomes)

- 'Formation de NO par décomposition radiolytique du nitroprussiate de sodium'. C. R. Acad. Sc. Paris., vol. 270, pp. 1.069-70. 1970 (com M. Cottin)

- 'Use of Mössbauer spectroscopy in the study of fossils'. Notas de Física, XVII, nำ 5. International Conf. On Xchists. Paraná, Brasil. 1971 (com E. Mattievich)

- 'Electron paramagnetic resonance and optical spectra of pentacyanocobaltate (II)'. J. Chem. Phys., vol. 54, pp. 3.760-9. 1971 (com F. D. Tsay e H. B. Gray)

- 'Mössbauer study of irradiated iron chelates and chemical consequences of ${ }^{57} \mathrm{Co}$ electron capture in complex ligand compounds'. J. Chem. Phys., vol. 56, pp. 1.269-74. 1972 (com E. Baggio Saitovitch e J. M. Friedt)

- 'Circular dichroism of hemoprotein derivatives in the visible region'. Chem. Phys. Lett., vol. 15, pp. 141-3. 1972 (com A. Garnier e J. Bolard)

- 'Applications of the Mössbauer effect in radiochemistry'. Radioch. Acta, vol. 17, pp. 173-90. 1972 (com J. M. Friedt)

- 'The role of water in the thermal and radiolytic reduction of alkalihexacyanoferrates (III)'. Radiochem. Radioanal. Lett., vol. 13, pp. 83-8. 1973 (com D. Raj)

- 'Étude par effect Mössbauer et rayons X des fluosilicates mixtes de fer et de zinc'. J. Phys. Colloq., vol. 35, pp. 87-9. 1974 (com F. Varret, P. Imbert e G. Jehanno)

- 'Covalency effects in hyperfine interactions in alkali dithioferrates'.J. Phys. Colloq., vol. 6, p. 241.1974 (com C. Taft e D. Raj)

- 'Mössbauer spectroscopy studies of thermal decomposition of alkali ferricyanides'. J. Inorg. Nucl. Chem., vol. 37, pp. 2.039-45. 1975 (com D. Raj) 
- 'Mössbauer spectra and electron exchange in tourmaline and staurolite'. J. Phys. Colloq., vol. 37, pp. 801-5. 1976 (com R. B. Scorzelli e E. Baggio Saitovitch)

- 'Mössbauer study of aging effects in ancient pottery from the mouth of the Amazon River'. J. Phys. Colloq., vol. 37, p. 866.1976 (com C. R. Enriques, E. Mattievich e M. C. M. Beltrão)

- 'Mössbauer spectroscopy of thermal and radiation effects in iron mineral and its applications to geochronology'. Proc. International Conf. on Mössbauer Spectroscopy, Bucareste, pp. 217-28. 1977 (com R. B. Scorzelli e H. Pollak)

- 'Thermoluminescent dating of archeological pottery from the Marajó Island (Brazil)'. Proc. of V International Conf. on Thermoluminescence Dosimetry. 1977 (com L. Courtois, M. C. M. Beltrão, J. L. Reyss, G. Valladas, M. F. Simão e H. Valladas)

- 'On superstructure in meteoritical taenite'. Meteoritics, vol. 13, p. 379. 1978 (com J. F. Albertsen, G. B. Jensen e J. M. Knudsen)

- 'Weathering model in paleomagnetic field intensity measurements on ancient fired clays'. Phys. of the Earth and Planetary Interiors, vol. 16, pp. 15-9. 1978 (com I. Sigalas e N. H. J. Gangas)

- 'Mössbauer and electron spin resonance study of electron irradiated ferrocyanide ions in KCl lattice'. J. Phys., vol. C 2, pp. 568-9. 1979 (com R. Scorzelli e N. V. Vugman)

- 'Microprobe observations on the Santa Catharina meteorite'. Anais da Academia Brasileira de Ciências, 51:1, pp. 181-2. 1979 (com H. D. Schorscher, C. M. Wiedeman, R. Scorzelli, I. Souza Azevedo e W. Curvello)

- 'Ressonância paramagnética eletrônica de sîlex'. Anais da Academia Brasileira de Ciências, vol. 51, p. 577. 1979 (com J. F. C. Marques, R. P. A. Muniz e M. C. M. Beltrão)

- 'Phase stability in iron-nickel invar alloys'. J. Phys., vol. 10, pp. L197-202. 1980 (com J. R. C. Guimarães, R. B. Scorzelli e I. Souza Azevedo)

- 'Observação de bactérias magnetotáticas no Rio de Janeiro'. Anais da Academia Brasileira de Ciências, vol. 53, p. 635.1981 (com D. M. S. Esquivel e H. G. P. Lins de Barros)

- 'Datação por termoluminescência de sílex do sítio arqueológico Alice Boer'. Anais da Academia Brasileira de Ciências. 1981 (com E. Zuleta, M. M. C. Beltrão, C. R. Enriquez e G. Poupeau)

- 'Biogenic magnetite in chlorophyceae algae'. Anais da Academia Brasileira de Ciências, vol. 54, pp. 599-600. 1982 (com D. M. S. Esquivel, H. G. P. Lins de Barros e R. B. Scorzelli)

- 'Electron microscopy and ultrastructure of a magnetotactic microorganism'. Biol. Cell., vol. 48, p. 1. 1983 (com M. Farina, H. G. P. L. Barros e D. M. S. Esquivel) 
- 'Les datations par résonnance paramagnétique électronique en géologie'. XXXIII Congrès de la Société Brésilienne de Géologie (resumo). 1984 (com G. Poupeau, A. Rossi e M. Teles)

- 'Solid state transformations in Fe-Ni alloys from meteorites in powder form'. Hyperfine Interact., vol. 28, pp. 979-83. 1986 (com R. B. Scorzelli e E. Galvão da Silva)

- 'A antiguidade do homem americano: novas perspectivas abertas pelo Projeto Central'. Revista do Instituto Histórico e Geográfico Brasileiro. 1988 (com M. C. Beltrão, S. Neme, F. A. Doria e C. O. C. Andrade)

- 'Analytical study of spherules from the site of the Tunguska (USSR) explosion'. C. R. Acad. Sci. Paris, vol. 308, pp. 1.589-95. 1989 (com C. Jehanno, D. Boclet, E. Robin e R. Rocchia)

- 'Cation distribution in natural ferrites'. Hyperfine Interact., vol. 77, pp. 43-50. (com H. A. Maia, F. F. T. Araujo, M. A. B. Araujo e R. B. Frankel)

- Bases físico-quimícas de la radioquímica. México, Comissão Nacional de Energia Nuclear. 1963

- Mössbauer effect and its applications in many body problems and other selected topics in theoretical physics. Nova York, Gordon and Breach. 1966

- 'Mössbauer spectroscopy'. Em H. A. O. Hill e P. Day (orgs.), Physical methods in advanced inorganic chemistry. Nova York, Interscience Publishers. 1968

- Lectures on the Mössbauer effect. Documents on Modern Physics Series. Nova York, Gordon and Breach. 1968

- ' $\mathrm{Fe}^{57} \mathrm{Metal}$, alloys and inorganic compounds'. Em V. I. Goldanskii e R. H. Herber (orgs.), Chemical applications of Mössbaner spectroscopy. Nova York, Academic Press. 1968 (traduzido para o russo em 1970)

- 'Applications to coordination chemistry'. Em L. May e Adam Hilger (orgs.), Introduction to Mössbauer spectroscopy. Londres. 1971

- 'Mössbauer spectroscopy'. Em E. Fluck e V. I. Goldanskii (orgs.), Modern Physics and Chemistry. Academic Press.

José Israel Vargas

- 'Influence of crystals defects on Szilard-Chalmers process in solids'. 1959 (com A. G. Maddock)

- 'Programme and utilization of the Triga reactor of the Instituto de Pesquisas Radioativas'. 1961 (com F. A. G. Gomes e M. Campos)

- 'The chemical effects of radiochemical neutron capture in solids'. 1961 (com A. G. Maddock)

- 'The use of tourmaline as a new thermal neutron dosimeter'. 1962 (com G. A. C. Tupynambá)

- 'Produção de ${ }^{51} \mathrm{Cr}$ de alta atividade específica'. 1962 (com M. Gonçalves de Alvarenga e E. A. Pimentel) 
- 'Efeitos de raio X sobre materiais ferro-elétricos'. 1963 (com C. Campi Murta)

- 'Radiochemical research at the Instituto de Pesquisas Radioativas'. 1963

- 'Chemical effects of radioactive neutron capture. Part 10: Influence of crystal defects on the retention'. 1963 (com A. G. Maddock e E. E. Treloar)

- 'Chemical consequences of thermal neutron capture in solids'. 1963

- 'The operation experience and the utilization of the Triga reactor'. 1963 (com M. Campos e O. C. Ferreira)

- 'Research in radiochemistry, physics and nuclear chemistry at the Instituto de Pesquisas Radioativas'. 1963

- 'Evidence of an after-effect in the (v. e. 36/56) reaction on polycrystalline hafnium (IV) acetyl-acetonate complex'. 1969 (com R. Beraud, I. Berkes, J. Daniere, M. Levy, G. Marest e R. Rougny)

- 'Applications of perturbed-angular correlations to the hot atom chemistry of hafnium 181 and selenium 75'. 1969 (com J. Berthier, P. Boyer e J. Oliveira)

- Applications of perturbed-angular correlations to the hot atom chemistry of hafnium 181 and selenium 75'. 1969 (com J. Berthier, P. Boyer e J. Oliveira)

- 'Effect of $(n, g)$ recoil on the g-g angular correlation in poly-crystalline Hf (IV) benzoil, phenyl-hydroxylamine complex'. 1969 (com J. Berthier, J. C. Hocquenghem, J. I. Robot e P. Boyer)

- 'Influence of gases and crystalline phase preparation on the isochronal annealing of copper phthalocyanine'. 1971 (com P. Odru)

- 'Influence des conditions d'irradiation et de l'état physique de l'échantillon sur la restauration des radicaux libres crées dans le pdichlorobenzene'. 1972 (com C. Dimitropoulos e H. Vargas)

- 'Free radicals formed by irradiation of 1-4 chlorobenzene'. 1972 (com C. Dimitropoulos)

- 'Differential perturbed angular correlation study of quadrupole coupling of ${ }^{181} \mathrm{Ta}$ in neutral octocoordinative d hafnium (IV) complexes'. 1972 (com P. Boyer, A. Tissier e P. Vullliet)

- 'The life-time variation of ${ }^{64} \mathrm{Cu}$ and the chemical state of $(\mathrm{n}, \mathrm{g})$ recoil copper in a copper phthalocyanine crystals'. 1972 (com P. Auric)

- 'The chemical consequences of ion implantation and thermal neutrons capture in alkali selenates'. 1972 (com M. Cogneau e G. Duplâtre)

- 'The chemical applications of angular correlation and half-life measurements'. 1972

- 'The variation of the life-time of ${ }^{89} \mathrm{Zr}$ in $\mathrm{BaTiO}_{3}, \mathrm{PbZrO}_{3}$ and $\mathrm{ZrO}_{2}$ '. 1974 (com P. Auric)

- 'Interação quadrupolar na fase cúbica estabilizada do $\mathrm{HfO}_{2}$ '. 1975 (com A. Baudry, O. de O. Damasceno, J. D. Fabris, J. R. F. Ferreira, J. Oliveira e A. L. Oliveira) 
- 'Estudo do tetramandelato de háfnio por correlação angular perturbada'. 1975 (com A. Baudry, O. de O. Damasceno, J. D. Fabris, J. R. F. Ferreira, A. L. Oliveira, J. Oliveira e L. I. Romanelli)

- 'Interações hiperfinas no hidreto de háfnio, estudadas por correlação angular perturbada'. 1976 (com A. Baudry, O. de O. Damasceno, J. D. Fabris, J. R. F. Ferreira, A. L. Oliveira, J. Oliveira e P. Boyer)

- 'Influência do pH do meio sobre a estabilidade de complexos de zircônio com o ácido mandélico'. 1976 (com H. N. S. Mansur, E. Mansur Netto e J. D. Fabris)

- 'Efeito da captura neutrônica e do annealing térmico sobre o tetramandelato de háfnio, medidos por correlação angular perturbada diferencial'. 1977 (com J. D. Fabris, A. Marques Netto e A. L. Baudry)

- 'Differential perturbed angular correlation measurements in reactor irradiated and thermal annealed tetrakis - (d-1-mandelate) hafnium (IV)'. 1978 (com J. D. Fabris, A. Marques Netto e A. L. Baudry)

- 'On the use of photoacoustic effect for investigating phase transition in solids'. 1980 (com C. Guizoni, M. A. Siqueira, L. C. Miranda e H. Vargas)

- 'Avaliação de demanda de energia do setor doméstico - Descrição e projeto de mercado - Abordagem metodológica'. 1989 (com C. Feu Alvim e Omar C. Ferreira)

- 'Perspectivas do setor de gás liqüefeito de petróleo frente ao aumento de participação de gás natural no mercado'. 1989 (com C. Feu Alvim e Omar C. Ferreira)

- 'Estado e ciência no Brasil'. Correio Brasiliense. 1994

- 'O desafio em ciência e tecnologia'. Correio Brasiliense. 1994

- 'A Internet brasileira na encruzilhada'. Jornal do Brasil. 1995

- 'Estímulo à tecnologia'. Jornal do Brasil. 1995

- 'Acordo espacial Brasil - China'. O Estado de S. Paulo. 1995

- 'Lei de patentes passa por um teste'. Gazeta Mercantil. 1996

- 'Lei de patentes'. Jornal de Brasília. 1996

- 'Núcleos de excelência'. Jornal do Brasil. 1996

- 'Política de ciência e tecnologia'. O Estado de S. Paulo. 1996

- 'Recursos humanos, empresas e tecnologia'. Correio Brasiliense. 1996

- 'Sobre os desafios da pós-graduação'. Jornal do Brasil. 1996

- 'A clonagem de mamíferos e biossegurança aética'. Gazeta Mercantil e outros. 1997

- 'How the North can help the South itself'. Nature. 1999

\section{Sergio Mascarenhas Oliveira}

De uma vasta relação bibliográfica, citam-se apenas alguns trabalhos publicados em revistas e livros abrangendo diferentes fases de sua vida profissional.

- 'O eletródio de antimônio e a solubilidade de $\mathrm{O}_{3} \mathrm{Sb}_{2}$ (rômbico) em soluções de HCl diluído'. Anais da Associação Brasileira de Química, vol. 11, pp. 95-8. 1952 (com E. O. M. Brandão e L. A. M. Carneiro) 
- 'Observation of the Costa Ribeiro effect in the process of sublimation of paraphenilene diamine'. Anais da Academia Brasileira de Ciências, 26:2, pp. 345-8. jun. 1954

- ' $\mathrm{CO}_{2}$ electrostatic generator'. Am. J. Phys., 26:8, pp. 563-6. Nov. 1958. Atas de Fis., no 8, pp. 563-6. 1961 (com F. Gambirasio e Y. P. Mascarenhas)

- 'Effect of nonuniform coloration on the power law relation between F - and F - aggregate - centers'. Phys. Stat. Sol., vol. 7, pp. 1.039-43. 1964 (com H. Rabin e V. Sverzut)

- 'Volume expansion and color center behavior of $\mathrm{NaCl}$ X-ray irradiated at low temperature'. Phys. Rev. A., 134:2, pp. 481-5. 1964 (com D. A. Wiegand e R. Smolughowski)

- 'Diffraction camara for precision measurements of lattice parameters at low temperatures'. Rev. Sci. Instr., 38:1, pp. 141-2. Jan. 1967 (com Y.P. Mascarenhas)

- 'Raman scattering from mixed $\mathrm{KBr}, \mathrm{KCl}$ crystals'. Phys. Lett. A., 26:5, pp. 194-5. Jan. 1968 (com J. P. Hurrell, S. P. S. Porto e T. C. Damen)

- 'Charge and polarization storage in ice crystals'. Em N. Riehl (org.), Physics of ice. Nova York, Plenum Press, pp. 483-91. 1969

- 'Rayleigh scattering in optically active liquids using a laser beam'. Bull. Am. Phys. Soc., 14:1, p. 74 . Jan. 1969 (com R. A. Carvalho e C. A. Arguello)

- 'EPR dosimetry of bones from the Hiroshima A-bomb site'. Bull. Am. Phys. Soc., 18:4, p. 579. 1973 (com A. Hasegawa e K. Takeshita)

- 'Denaturation horn keratin observed by piezoelectric measurements'. Bioch. Biophys. Res. Comm., vol. 62, pp. 415-8. 1975 (com E. Fukada e R. L. Zimmerman)

- 'Electrical effects during condensation and phase transitions of ice'. J. Chem. Phys., 68:8, pp. 3.823-8. Apr. 1978 (com L. Onsager e D. L. Staebler)

- 'Bioelectrets: electrets in biomaterials and biopolymers'. Em G. M. Sessler (org.), Electrets. Nova York, Springer Verlag, pp. 321-46. Topics in applied physics, 33. 1979

- 'A correlated walk model for therally stimulated dipolarization currents in keratin', J. Chem. Phys., 76:11, pp. 5.643-5. June 1982 (com E. Blaisten-Barojas)

- 'Photoacoustical radiation dosimeter'. Med. Phys. 11:1, pp. 73-4. Jan.-Feb. 1984 (com H. Vargas e C. L. Cesar)

- 'Experimental model of electric simulation of pseudoarthrosos healing'. Clin. Orth. Rel. Res., no 183, pp. 267-75. Mar. 1984 (com A. E. Rodrigues Fuentes, J. P. M. de Souza e V. Valeri)

- 'ESR dating of shells from sambaquis (Brazilian shell mounds)'. Em M. Ikeya e T. Miki (orgs.), ESR dating and dosimetry. Tóquio, Ionics, pp. 139-43. 1985 (com O. Baffa) 
- 'Proton NMR relaxation of hydrated insulin powder'. Atomindex, 18:6, p. 2.495. 1985 (com R. Sanches, J. P. Donoso e H. Panepucci)

- 'Transient soil-water infiltration using X-ray CAT scanner'. EOS Transactions, American Geophysical Union, 69:4, p. 1.220. Nov. 1988 (com S. Crestana, D. R. Nielsen e R. Pozzi-Mucelli)

- 'Two thermal methods to measure the energy fluence of a brief exposure of diagnostic X-rays'. Medical Physics, 19:3, pp. 575-7. May-June 1992 (com A. A. Carvalho, M. H. de Paula e J. R. Cameron)

- 'Nuclear technique in soil science'. Trends in Agricultural Sciences. Soil Science, vol. 1, pp. 27-46. 1993 (com R. Cesareo e S. Crestana)

- 'Interdisciplinaridade em ciência e tecnologia'. Ciência \& Engenharia, 3:1, pp. 5-6. jan.-jul. 1994

- Instrumentação agropecuária: contribuições no limiar do novo século. Brasilia, Embrapa/SPI. 1996 (Além de ser co-editor desta obra, Sergio Mascarenhas participou da elaboração de alguns de seus capítulos.)

\section{Yvonne Primerano Mascarenhas}

Especialista em cristalografia estrutural por difração de raios X, Yvonne Mascarenhas desenvolveu trabalhos nas seguintes linhas de pesquisa: determinação de estrutura de pequenas moléculas usando difração de raios X por monocristais; análise estrutural de macromoléculas em solução por espalhamento de raios X; determinação de estrutura molecular de proteínas; caracterização estrutural de materiais policristalinos de interesse tecnológico. Entre cerca de oitenta artigos publicados em revistas especializadas, a autora destaca os cinco primeiros listados a seguir.

- 'The crystal structure of perdeuterated violuric acid monohydrate, by the X-ray diffraction analysis'. Acta Cryst., no 17, p. 407.1964 (com B. M. Craven)

- 'Crystal structure analysis of deaminooxytocin: conformational flexibility and receptor binding'. Science, 232:4.750, pp. 633-6. May 21986 (com S. P. Wood, I. J. Tickle, A. M. Theharne, J. E. Pitts, J. Y. Li, J. Husain, S. Cooper, T. Blundell, V. J. Hruby, A. Buku, A. J. Fischman e H. R. Wyssbrod)

- 'Location of cerium and lanthanum cations in Ce Na Y and La Na Y after calcination'. Zeolites, vol. 18, pp. 44-9. 1997 (com J. G. Nery, T. J. Bonagamba, N. C. Mello e E. F. Souza-Aguiar)

- 'Crystallographic and spectroscopic characterization of a molecular hinge: conformational changes in bothropstoxin 1 , a dimeric lys49phospholipase A2 homologue'. Proteins: structure, function and genetics, no 30, pp. 442-54. 1998. (com M. T. S. Giotto, R. C. Garratt, G. Oliva, J. R. Giglio, A. C. O. Cintra, W. F. Azevedo Jr., R. K. Arni e R. J. Ward) 
- 'The structure of orthorhombic hafniates by neutron powder diffraction and perturbed-angular-correlation spectroscopy (PAC)'. Mat. Sci. Forum, vol. 278-181, pp. 720-5. 1998 (com J. A . Guevara, S. F. Cuffini, R. E. Carbonio, J. A . Alonso, M. T. Fernandes, P. de La Presa, A. Ayala e A. López Garcia)

- 'X-ray analysis of deaminooxytocin: conformational flexibility and receptor binding'. Em R. M. Bernet e H. J. Vogel (orgs.), Biological organization: macromolecular interactions at high resolution. Nova York, Academic Press, pp. 289-306. 1987 (com J. E. Pitts, S. P. Wood, I. J. Tickle, A. M. Theharne, J. Y. Li, J. Husain, S. Cooper e T. L. Blundell)

- 'Refinamento de estruturas cristalinas de macromoléculas por dinâmica molecular'. Conferências e cursos da VI Escola LatinoAmericana de Química Teórica (ELAQT). 1988

- 'CO $\mathrm{CO}_{2}$ electrostatic generator'. Am. J. Phys., 26:8, pp. 563-6. Nov. 1958. Atas de Fis., nº 8, pp. 563-6. 1961 (com S. Mascarenhas e F. Gambirasio)

- 'X-ray diffraction studies of the Hg-In alloy system'. J. Appl. Crystal, 3:5, pp. 294-6. Oct. 1970

- 'Crystallographic studies of a serie of phenyl and benzyl phosphine oxides $\mathrm{CoCl}_{2}$ complexes'. Anais da Academia Brasileira de Ciências, 47:2, pp. 227-30. 1975 (com K. Tomita)

- 'Crystalline and molecular structure of aniba-dimer A'. Anais da Academia Brasileira de Ciências, 49:1, pp. 119-26. 1977 (com O. R. Gottlieb)

- 'The crystal and molecular structure of bis (tribenzylphosphine oxide) dichloro cobalt (II)'. J. Coord. Chem., no 9, pp. 59-64. 1979 (com R. H. de Almeida Santos)

- 'Sonderianin, a new furanoid diterpene croton sonderianus Muell. Arg.'. Phytochemistry, 20:4, pp. 852-4. 1981 (com A. A. Craveiro, E. R. Silveira e R. Braz Filho)

- 'Structure and conformational changes in crotamine'. Arch. Biol. Med. Exp., 16:2, p. R-139. 1983 (com J. R. Beltram, H. Cid e A. F. Craievich)

- 'Saxs study of structure and conformational change of crotamine'. Biophys. J., 47:1, pp. 33-5. Jan. 1985 (com J. L. Beltran, A. F. Craievich e C. J. Laure)

- 'Methyl and phenylmercury(II) derivatives of 2mercaptobenzothiazole. Crystal structure of (2-mercaptothiazolate) methylmercury(II)'. Inorg. Chem., 24:21, pp. 3.435-8. 1985 (com J. Bravo, J. S. Casas, M. V. Castano, M. Gayoso, A. Sanches, C. O. P. Santos e J. Sordo)

- 'Crystal structures of bis-11-aminoundecanoic chlorocuprate and bis11-aminoundecanoic-Cu(II) sulfate trihydrate'. J. Crystallogr. Spectrosc. Resc., 17:5, pp. 633-52. 1987 (com I. Vencato e L. Tosi) 
- 'Structure of 5-hydroxy-2,2-dimethyl-4-(p-toluenesulfonamido)3-hexanone'. Acta Crystallogr. C., 45:8, pp. 1.174-6. Aug. 1989 (com I. Vencato, R. A. Pilli e M. I. H. Agostini)

- 'Molecular and crystal structure of 2-bromo-1-hydroxy-1-phenyl4, 4-dimethyl-3-pentanone'. J. Braz. Chem. Soc., 1:2, pp. 59-62. 1990 (com I. Vencato, R. A. Pilli e N. M. C. Grimald)

- 'Structure-function relationship for the highly toxic crotoxin from Crotallus durissus terrificus'. Eur. Biophys. J., vol. 21, pp. 199-205. 1992 (com P. F. W. Stouten, J. R. Beltran, C. J. Laure e G. Vriend)

- 'Phase characterization of lead zirconate titanate obtained from organic solutions of citrates'. J. Am. Ceram. Soc., 75:8, pp. 2.088-93. 1992 (com M. A. Zaghete, C. O. P. Santos, J. A. Varela e E. Longo)

- 'Pore structure characterization of kaolin and their acid-treated products using small-angle X-ray scattering'. Appl. Clay Sci., vol. 8, pp. 397-404. 1994 (com D. R. Vollet e J. C. D. Macedo)

- 'Laser heated pedestal growth of orthorhombic $\mathrm{SrHfO}_{3}$ single crystal fiber'. J. of Crystal Growth, no 200, pp. 621-4. 1999 (com M. R. B. Andreeta, A. C. Hernandes, S. F. Cuffini e J. A. Guevara)

\section{Milton Ferreira de Souza}

- 'Thermal conduction of liquid dielectric under electric fields (fatty acids)'. Anais da Academia Brasileira de Ciências, 28:1, pp. 96-8. 1956 (com S. Mascarenhas, Y. P. Mascarenhas e R. F. Rabello)

- 'Measurement of small dc potencials from low power sources'. Amer. J. Phys., 26:3, pp. 193-4. 1958 (com S. Mascarenhas)

- 'Effective volumes of vacancies of divalents ions and their diffusion coefficient in alkali halides'. Phys. Rev., 188:3, pp. 1.367-70. 1969

- 'Polarized local mode absorption of aligned $\mathrm{H}^{-}-\mathrm{H}^{-}, \mathrm{D}^{-}-\mathrm{D}^{-}$and $\mathrm{H}^{-}-\mathrm{D}^{-}$ pairs in alkali halides'. Phys. Rev. Let., 26:20, pp. 1.426-30. 1970 (com A. D. Gongora, M. Aegerter e F. Luty)

- 'Dielectric relaxation of $\mathrm{Ca}^{+2}$ vacancy dipoles in $\mathrm{KCl}+\mathrm{KBr}$ mixed crystals'. Phys. Stat. Sol., B59, pp. 335-41. 1973 (com R. Barbosa e G. F. L. Ferreira)

- 'Off center $\mathrm{Cu}^{+}$ions in potassium halides studied with ionic thermocurrents'. Phys. Rev., 7:10, pp. 4.677-82. 1973 (com M. Siu Li e F. Luty)

- 'Infrared absorption of pairs of coupled local-mode oscillators: H-H, D-D and H-D pairs in KCl'. Phys. Rev., 8:12, pp. 5.866-74. 1973 (com F. Luty)

- 'Interaction between F centers and interstitial $\mathrm{H}$ atoms in $\mathrm{KCl}$ crystals'. Phys. Rev., 10:4, pp. 1.617-22. 1974 (com V. R. Dumke)

- 'Local modes of $\mathrm{H}^{-}-\mathrm{H}^{-}, \mathrm{D}^{-}-\mathrm{D}^{-}$and $\mathrm{H}^{-} \mathrm{D}^{-}$pairs in $\mathrm{KBr}, \mathrm{KI}, \mathrm{NaCl}$, and RbCl'. Phys. Rev., 9:12, pp. 5.257-63. 1974 (com R. Robert)

- 'A model for the interaction between F centers and $\mathrm{H}$ atoms in ionic crystals'. Rev. Bras. Fis., 5:2, pp. 201-6. 1975 (com V. R. Dumke) 
- 'Local mode of $\mathrm{H}^{-}$substitutional ion in KCN'. Phys. Stat. Sol., 77:2, pp. 685-7. 1976 (com J. C. Castro e H. C. Basso)

- 'The dielectric relaxation of impurity-vacancy complexes in KI doped with Cl- anions'. Phys. Stat. Sol., 80:2, pp. 597-602. 1978 (com S. C. Zilio)

- 'The ordering of aqueous suspensions of polysterine spheres'. Sol. Stat. Com., 35:12, pp. 907-10. 1980 (com M. K. Udo)

- 'Ionic thermal currents under uniaxial stress: the elastic dipole moment'. Phys. Stat. Sol., 112:2, pp. 693-7. 1982 (com M. Siu Li e S. E. Kapphan)

- 'Construção e calibração de radiômetros solares: piranômetro e piroeliômetro'. Rev. Fis. Apl. Instrum., no 3, pp. 118-33. 1988 (com J. F. Escobedo e E. F. Passos)

- 'Impure zirconia electrical conductivity enhancement by rare earth minority ions in the $\mathrm{Y}_{2} \mathrm{O}_{3}: \operatorname{Re}_{2} \mathrm{O}_{3}: \mathrm{ZrO}_{2}$ system'. J. Mater. Sci., no 30 , pp. 4.355-62. 1995 (com D. P. F. de Souza e A. F. Chinelatto)

- 'Alumina purification by carbothermal reduction'. J. Mater. Chem., 6:2, pp. 233-8. 1996 (com D. P. F. de Souza)

- 'Grain boundary conductivity of zirconia yttria and the space charge concept'. Radiation Effects and Defects in Solids, nำ 147, pp. 215-27. 1998 (com D. M. P. F. de Souza)

- 'On the up conversion emission of rare earth doped zirconia'. Radiation Effects and Defects in Solids, n 147, pp. 77-81. 1998 (com F. S. Vicente, A. C. Hernandes, M. A. P. Andreeta e M. Siu Li)

- 'Glass phase expelling during liquid phase sintering'. Materials Research, no 11, pp. 53-8. 1998 (com D. P. F. de Souza)

- 'Preparação de mulita a partir de hidróxido de alumínio e sílica da casca de arroz'. Cerâmica, 45:291, pp. 33-7. 1999 (com J. Yamamoto)

- 'Microstructure, electric and magnetic properties to improve the ceramic processing of strontium doped lanthanum manganate'. Acta Microscopica, supl. A, pp. 173-6. 1998 (com R. A. Mesquita e D. P. F. de Souza)

\section{Horacio Cintra de Magalhães Macedo}

A bibliografia de Horacio Macedo envolve sobretudo a publicação de livros didáticos de física, físico-química e química.

- Termodinâmica estatística. São Paulo, Edgar Bluecher. 1975 (com Adir M. Luiz)

- Dicionário de Física. Rio de Janeiro, Nova Fronteira. 1976

- Termodinâmica básica. São Paulo, Edgar Bluecher. 1976 (com Adir M. Luiz)

- Elementos da teoria cinética dos gases. Rio de Janeiro, Guanabara Dois. 1978

- Físico-química I. Rio de Janeiro, Guanabara Dois. 1980 
Traduções e adaptações

- Cotton e Lynch. Curso de química. Rio de Janeiro, Forum. 1970

- Cotton e Lynch. Curso de química, guia do mestre. Rio de Janeiro, Forum. 1970

- História da ciência. Rio de Janeiro, Difusão Européia do Livro, vol. XI. 1972 (com P. Sussekind Rocha)

- Crockford. Físico-química básica. Rio de Janeiro, LTC — Livros Técnicos e Científicos. 1977

- Cotton e Wilkinson. Química fundamental. Rio de Janeiro, LTC Livros Técnicos e Científicos. 1978

- P. Tippler. Física. 1aㅡ ed., Rio de Janeiro, Guanabara Dois. 1978 (A tradução desta obra já se encontra na $4^{a}$ edição, sendo a última de 2000, que contou com a colaboração de Ronaldo de Biasi e foi editada por LTC - Livros Técnicos e Científicos, Rio de Janeiro.)

- Perry e Chilton. Manual do engenheiro químico. Rio de Janeiro, Guanabara Dois. 1979 (com P. E. Barbosa e Luiza Maria Barbosa).

- Smith e Van Ness. Termodinâmica da engenharia química. Rio de Janeiro, Guanabara Dois. 1979

- Shreve e Brink. Indústrias dos processos químicos. Rio de Janeiro, Guanabara Dois. 1980

- Foust. Princípios das operações unitárias. $2^{a}$ ed., Rio de Janeiro, Guanabara Dois. 1981

- Jackson. Eletromagnetismo clássico. $3^{\mathrm{a}}$ ed., Rio de Janeiro, Guanabara Dois. 1982 (com A. Macedo).

- Faires e Simang. Termodinâmica. 6a ed., Rio de Janeiro, Guanabara Dois. 1983

- Shriner. Identificação dos compostos orgânicos. Rio de Janeiro, Guanabara Dois. 1983

- Vogel. Análise inorgânica quantitativa. $5^{a}$ ed., Rio de Janeiro, Guanabara Koogan. 1990

- M. N. Ozisik. Transferência de calor. 5르. ed., Rio de Janeiro, Guanabara Koogan. 1991

- F. P. Incropera e D. P. de Witt. Fundamentos de transferência de calor e de massa. 3a ed., Rio de Janeiro, Guanabara Koogan. 1992

- W. E. Boyce e F. C. Diprima. Equações diferenciais elementares e problemas de valores de contorno. $4^{\mathrm{a}} \mathrm{ed}$., Rio de Janeiro, Guanabara Koogan. 1994

- Kotz e Treichel. Química e reações químicas. $3^{\mathrm{a}}$ ed., Rio de Janeiro, LTC - Livros Técnicos e Científicos, 2 vols. 1998

- P. W. Atkins. Físico-química. $6^{\mathrm{a}}$ ed., Rio de Janeiro, LTC — Livros Técnicos e Científicos, 3 vols. 1999

Cabe mencionar que Horacio Macedo prestou colaboração especializada, nas áreas de matemática, estatística, física, físico-química e química, na eleboração do Novo Dicionário da Lingua Portuguesa de 
Aurelio Buarque de Holanda Ferreira (Rio de Janeiro, Nova Fronteira). Acrescentamos também que o currículo de Jacques Danon (versão encontrada na Academia Brasileira de Ciências) apresenta o trabalho 'A stochastic theory of chromatography' (Notas de Física, vol. IV, no 7, 1958), realizado em colaboração com H. Macedo e A. A. L. Zamith.

Paulo Emidio de Freitas Barbosa

- 'Espetrografia qualitativa e semiquantitativa: limite de identificação', Anais da Associação Química do Brasil, vol. VI. 1947

- 'A identificação do potássio com a dipicrilamina'. Revista da Sociedade Brasileira de Química, vol. X, no 4. 1941

- 'A identificação de compostos ácidos, solúveis e insolúveis'. Boletim do Laboratório da Produção Mineral, no 5.

- 'A new procedure for detecting acidity'. Industrial and Engineering Chemistry - Analytical Edition, 14:6 (com Fritz Feigl)

- 'Dosagem de berílio pela 8-oxiquinoleína'. Boletim do Laboratório da Produção Mineral, no 17. 1945

- 'Espetrografia quantitativa - Método da energia total'. Boletim do Laboratório da Produção Mineral, no 18.

- 'Estudo sobre condições da precisão em espetrografia quantitativa'. Anais da Associação Química do Brasil, vol. IX. 1949

- 'Um método para aumentar a precisão de análises espectrográficas quantitativas'. Anais da Associação Química do Brasil, vol. IX. 1949

- 'Sugestões sobre calibração de chapas em fotometria fotográfica'. Boletim do Laboratório da Produção Mineral, no 35.1949

- Deslocamento isotópico de massa em espetros atômicos. Tese de concurso para livre-docente de física, Rio de Janeiro, Universidade do Brasil. 1952

- Redes de difração e suas montagens. Tese de concurso para a cátedra de física, Rio de Janeiro, Universidade do Brasil. 1957

- 'Radioatividade'. Enciclopédia técnica universal. Rio de Janeiro, Globo, vol. I.

- 'Métodos físico-químicos em análise química'. Química, I:1. 1945

- 'Análise isotópica por espetroscopia'. Ciência e Cultura. mar. 1954

- 'O comércio de rutilo no Ceará'. Boletim do Ministério da Agricultura. 1942

- 'Universidade: autarquia ou fundação?'. Jornal do Brasil. Caderno Especial. 17.6.1968

- 'A organização jurídica da universidade brasileira'. Cadernos Brasileiros, ano XI, nº 52. abr. 1969

Apostilas

- 'Lições de termodinâmica'. Conselho Nacional do Petróleo. 1952

- 'Espectroscopia experimental'. Departamento de Química da Faculdade Nacional de Filosofia da UB. 1952 
- 'Espectros atômicos e moleculares'. Departamento de Química da Faculdade Nacional de Filosofia da UB. 1952

- 'Práticas de ótica'. Centro Brasileiro de Pesquisas Físicas. 1956

- 'Práticas de eletricidade e magnetismo'. Centro Brasileiro de Pesquisas Físicas. 1953

- 'Introdução à termodinâmica química'. Conselho Nacional do Petróleo. 1954

- 'Medidas elétricas - manual de laboratório'. Escola de Química da UFRJ. 1961

- 'Termodinâmica do equilíbrio físico'. Petrobras: curso de refinação de petróleo. 1964

- 'Produção de energia: fundamentos termodinâmicos dos processos de produção de energia. 1964

- 'Refrigeração: fundamentos termodinâmicos dos processos de refrigeração. 1964

\section{Bartyra de Castro Arezzo}

- 'Observações sobre a determinação quantitativa de tório em areias monazíticas'. Revista da Sociedade Brasileira de Química, vol. XVIII, $\mathrm{n}^{\mathrm{o}} 3-4.1948$

- 'Radiochemical measurements of $14 \mathrm{MeV}$ cross-section reactions of V-51'. Durham, Londondery Laboratory for Radiochemistry, Durham College, University of Durham. 1948-49

- 'Sobre o mecanismo de extração do nitrato de escândio por solventes orgânicos'. Anais da Academia Brasileira de Ciências, 32:3-4. dez. 1960

- 'Extraction of gallium from hydrochloric acid into mixed organic solvent'. Progress Report, Laboratory for Nuclear Science, MIT. Nov. 1은 1961

- 'Solvent extraction of scandium'. Proceedings da Primeira Conferência Interamericana de Radioquímica, Montevidéu. July 1963 (com J. Danon)

- 'The annealing of $(\mathrm{n}, \mathrm{y})$ recoil damage in ferroelectric triglycine sulphate'. Proceedings of Symposium on Chemical Effects Associated with Nuclear Reactions and Radioactive Transformations, IAEA. Dec. 1964 (com J. I. Vargas)

- 'Conseqüências físico-químicas da captura radiante de nêutrons térmicos no sulfato hexahidratado de guanidina'. Resumos da XVII Reunião da Sociedade Brasileira para o Progresso da Ciência, Belo Horizonte. jul. 1965 (com J. I. Vargas)

- 'Contribuição ao estudo dos metais trivalentes na extração por solventes: sistema HCl-Ga(III)-Nitrobenzeno-Benzeno'. Tese de livredocência e doutoramento, Rio de Janeiro, Universidade do Estado da Guanabara. 1963 
- 'Transformações alotrópicas no selênio investigadas com correlações angulares gama-gama'. Resumos da XXIII Reunião da Sociedade Brasileira para o Progresso da Ciência, Salvador. jul. 1970 (com D. Binns e A. Marques)

- 'Electronegativity effects in T-for-CH2-X substitution for recoil tritium reactions with n-propyl fluoride'. J. of Phys. Chem., no 76, p. 187. 1972 (com Thomas Smail e F. S. Rowland)

- 'Pesquisas em química nuclear e radioquímica'. Resumos da XVIV Reunião da Sociedade Brasileira para o Progresso da Ciência, São Paulo. jul. 1972 (com Alfredo V. Bellido)

- 'Análise não destrutiva de traços de elementos em carvões com ativação com nêutrons de ressonância'. Resumo do I Encontro Nacional de Química Analítica, Rio de Janeiro, Departamento de Química, Pontifícia Universidade Católica, 18-19. 11. 1982 (com L. F. Bellido)

- 'Determinação de traços de impurezas em hélio por cromatografia em fase gasosa'. Resumos do I Encontro Nacional de Química Analítica, Rio de Janeiro, Departamento de Química, Pontifícia Universidade Católica, 18-19.11.1982 (com L. Cosme Tadeu)

- 'Análise de urânio e tório em cinzas e volantes de usinas termoelétricas brasileiras'. Resumo do II Encontro Nacional de Química Analitica, Rio de Janeiro, Departamento de Química, Pontifícia Universidade Católica, 9-11.11. 1983 (com L. F. Bellido)

- 'Determination of radioactive trace elements in ashes and fly-ashes from Brazilian coal-fired power plants'. Proceedings of the Fifth International Conference on Nuclear Methods in Enviromental and Energy Research, Porto Rico. 2-6.4.1984 (com L. F. Bellido)

- 'Uranium and thorim determination in Brazilian coal by epithermal neutron activation analysis'. J. Radioanal. Nuc. Chem., vol. 92-1, pp. 151-8. 1985 (com L. F. Bellido)

- 'Non-destructive analysis of inorganic impurities in Brazilian coals by epithermal neutron activation'. J. Radioanal. Nuc. Chem., vol. 100-1, pp. 21-9. 1986 (com L. F. Bellido)

- 'Análise não destrutiva de traços de elementos em carvão mineral por ativação com nêutrons de ressonância'. Química Nova, vol. 101, pp. 42-3 (comunicação). 1987 (com L. F. Bellido)

\section{Silvia Tiomno Tolmasquim}

- 'Algumas aplicações industriais da sacarose - parte I'. Revista Científica, Rio de Janeiro, 1:4, pp. 23-30. 1950

- 'Algumas aplicações industriais da sacarose - parte II'. Revista Científica, Rio de Janeiro, 2:1-2, pp. 43-51. 1951

- 'Purificação da amilopectina em coluna de polpa de celulose'. Anais da Academia Brasileira de Ciências, 33:3-4, pp. 305-7. 1961 (com F. R. T. Rosenthal e E. Tolmasquim) 
- 'A posição da química na escola secundária'. Escola Secundária, no 16, pp. 84-6. 1961. Publicação da Campanha de Aperfeiçoamento e Difusão do Ensino Secundário (CADES/MEC).

- 'Contribuição ao estudo da cinética da reação do amido com o ácido monocloroacético e o anidrido acético'. Tese de concurso, Rio de Janeiro, Faculdade de Filosofia, Ciências e Letras, Universidade do Estado da Guanabara. 1963

- 'Estudo do amido de grão-de-bico comercial com o viscoamilógrafo Brabender'. VI Reunião Anual da Divisão de Química Orgânica e Bioquímica da Associação Brasileira de Química, Rio de Janeiro, pp. 17-8 (resumo). 1964 (com E. Tolmasquim)

- 'Traçadores radioativos e suas aplicações químicas'. Boletim do Curso de Introdução à Radiobiologia, IV Curso de Introdução à Radiobiologia, Instituto de Biofísica da Escola de Medicina e Cirurgia do Rio de Janeiro e Comissão Nacional de Energia Nuclear, pp. 3943. 1964.

- 'A study of chick-pea (Cicer arietinum) starch. I - Brabender viscosity'. Anais da Academia Brasileira de Ciências, 37:2, pp. 2359. 1965 (com M. A. Zamith, F. R. T. Rosenthal e E. Tolmasquim)

- 'Química-acontecimentos de 1966'. Anuário da Enciclopédia DeltaLarousse. Rio de Janeiro, Delta, pp. 572-5. 1967 (com E. Tolmasquim)

- 'New starches: properties of five varieties of cow-pea (Vigna sinensis) starch'. Reunião Conjunta de Abril da American Association of Cereal Chemists e American Oil Chemists Society. Washington D. C. 1968 (com E. Tolmasquim e A. M. N. Corrêa)

- 'Química - acontecimentos de 1967’. Anuário da Enciclopédia DeltaLarousse. Rio de Janeiro, Delta, pp. 584-7. 1968 (com E. Tolmasquim)

- 'Química - acontecimentos de 1968'. Anuário da Enciclopédia DeltaLarousse. Rio de Janeiro, Delta. 1969 (com E. Tolmasquim)

- 'New starches. Properties of five varieties of cowpea starch'. Cereal Chemistry, Publicação da American Association of Cereal Chemists, Inc., 48:2, pp. 132-9. 1971 (com E. Tolmasquim e A. M. N. Corrêa)

- 'Química - eventos de 1973'. Livro do Ano da Enciclopédia Século XX. Rio de Janeiro, Expressão e Cultura, pp. 248-9. 1974

- 'Programa de capacitação de recursos humanos'. Informativo do INT, IX:12, pp. 15-8. 1976

- 'Carotenos e energia radiante'. Informativo do INT, X:14, pp. 20-8. 1977 (com A. M. P. Gallotti)

- Subsídios do INT à análise da evolução e perspectivas do desenvolvimento brasileiro. Rio de Janeiro, Instituto Nacional de Tecnologia, 66p. 1978.

- 'Alternativas energéticas de origem vegetal - situação brasileira'. Rio de Janeiro, Fórum de Ciência e Cultura/UFRJ, IX Curso de Estudos de Problemas Brasileiros, 46p. 1979.

- 'Treinamento de recursos humanos para o desenvolvimento tecnológico do país. Balanço de experiências'. $4^{\circ}$ Congresso Brasileiro 
Agradecimento As autoras registram aqui seus agradecimentos a todos que facilitaram 0 acesso às fontes de dados: Mast/Arquivo de História da Ciência, Academia Brasileira de Ciências/ Projeto Memória, INT/Memória Técnica, Professoras Doutoras Aida Espinola (COPPE) e Elza Vasconcelos (UNICAMP), cientistas citados, seus colaboradorese familiares. de Treinamento e Desenvolvimento. Rio de Janeiro, Associação Brasileira de Treinamento e Desenvolvimento (ABTD)/Fundação de Amparo à Pesquisa do Estado do Rio de Janeiro (Faperj) (resumo). 1982

- 'Capacitação de recursos humanos para o desenvolvimento tecnológico'. Informativo do INT, XV:30, pp. 39-42. 1982

- 'O INT e a capacitação de recursos humanos na área tecnologia meio ambiente'. Seminário Latino-Americano sobre Tecnologia e Meio Ambiente. Rio de Janeiro, Programa das Nações Unidas para o Meio Ambiente (PNUMA)/Rede de Formação Ambiental para a América Latina e o Caribe (Ucored), 13p. 1982

- 'Estudos brasileiros - Abstracts'. Rio de Janeiro, Fórum de Ciência e Cultura/UFRJ, 60p. 1986

- 'Equilíbrio químico'. Curso para aperfeiçoamento de professores de ensino secundário. Rio de Janeiro, Faculdade Nacional de Filosofia, UB, 12p. 1956

- 'Práticas de físico-química'. Cadeira de físico-química e química superior. Rio de Janeiro, Faculdade Nacional de Filosofia, UB, 91p. 1957

- Química (30 ano científico). $1^{\text {a }}$ e 2a ed., 220p. 1959 (com E. Tolmasquim)

- 'Práticas de eletroquímica'. Cadeira de físico-química e química superior. Rio de Janeiro, Faculdade Nacional de Filosofia, UB, 37p. 1964

- 'Estrutura atômica (para o curso colegial)', 19p. 1968 (com E. Tolmasquim)

\section{NOTAS}

${ }^{1}$ Acreditamos que Costa Ribeiro quisesse, na realidade, se referir a Alberto Betim Paes Leme (professor, pesquisador e diretor do Museu Nacional).

${ }^{2} \mathrm{O}$ grande entrosamento de Vargas com a comunidade científica do Rio de Janeiro justifica a inclusão de seu nome em uma relação local, apesar de ele estar vinculado ao estado de Minas Gerais.

${ }^{3}$ Mabel Rodrigues defendeu a primeira tese de doutoramento no campo da química (1968), em São Carlos.

${ }^{4}$ Ricardo de Carvalho Ferreira é químico pela Universidade de São Paulo (USP) (1946-50) e pela Universidade Católica de Pernambuco (UCPE) (1951-52) (Schwartzman, 1979, p. 460). José Leite Lopes é químico industrial pela Escola de Engenharia de Pernambuco (1936-39). Tendo vindo ao Rio de Janeiro, em 1937, para o $3^{\circ}$ Congresso Sul-Americano de Química, teve oportunidade de conhecer Mario Schemberg, também pernambucano, que acabara de concluir o curso de física, na USP. Leite Lopes voltou para Recife, decidido a estudar física, mas resolveu concluir o curso de química industrial. Recebeu uma bolsa de estudos para o Rio de Janeiro, mas, nesta cidade, em vez de se especializar em química, fez exame vestibular para o curso de física da FNFi/UB (1940) (ver Leite Lopes, 1992, pp. 289-90).

${ }^{5}$ Cardoso escolheu o amido para tema inicial dos estudos, por se tratar de matéria-prima abundante e barata no Brasil.

${ }^{6}$ Para não se tornarem exaustivamente longas, as citações bibliográficas não pretendem ser completas, mas apenas rastrear as diferentes áreas de trabalho dos personagens citados. Indicamos, a seguir, as respectivas fontes de dados:

- José Carneiro Felippe: Serra Costa, op. cit., pp. 30-1;

- João Christovão Cardoso: fontes indicadas no texto;

- Augusto Araujo Lopes Zamith: currículo de Jacques Danon;

- Alberto Betim Paes Leme: Iglesias, Dolores et al. 'Bibliografia e índice da geologia do Brasil - 1641-1940'. Boletim do Laboratório da Produção Mineral, n² 204, pp. 231-2. 1959;

- Ernesto Lopes da Fonseca Costa: bibliografia elaborada pelo INT/Memória Técnica;

- Alvaro Alberto da Mota e Silva: bibliografia elaborada pela Academia Brasileira de Ciências/Projeto Memória; 
- Hans Ernst Werner Zocher: bibliografia elaborada pela Academia Brasileira de Ciências/Projeto Memória;

- Hervasio Guimarães de Carvalho: currículo;

- Sergio Pereira da Silva Porto: currículo;

- Jacques Abulafia Danon: currículo;

- José Israel Vargas: currículo;

- Sergio Mascarenhas Oliveira: currículo;

- Yvonne Primerano Mascarenhas: currículo;

- Milton Ferreira de Souza: currículo;

- Horácio Cintra de Magalhães Macedo: arquivo pessoal da professora dra. Aida Espinola (COPPE/UFRJ);

- Paulo Emídio de Freitas Barbosa: currículo;

- Bartyra de Castro Arezzo: currículo;

- Silvia Tiomno Tolmasquim: currículo;

7 Em seu 'Regulamento', publicado em 1950, a FNFi esboçou uma proposta de curso de doutoramento que não chegou a se concretizar.

\section{FONTES PRIMÁRIAS}

Ciornai, leuda 1982

Professores do

Departamento de

Físico-Química

S. d.

Rodrigues, Edson et al. 1998
'Memorial: progressão vertical de professor assistente prevista no decreto n 85487/80'. Rio de Janeiro, Escola de Química, UFRJ.

'João Christovão Cardoso'. Rio de Janeiro, Instituto de Química/UFRJ. (Homenagem postuma)

'Breve história da implantação da química em São Carlos'.

Trabalho apresentado no Congresso da USP de História da Ciência, São Paulo.

\section{REFERÊNCIAS BIBLIOGRÁFICAS}

Costa Ribeiro, Joaquim 1994

Daniels, Farrington et al. 1958

Leite Lopes, José 1992

Macedo, Horacio 1976

Mooradian, Aram 1979

Motoyama, Shozo 1979

Muniz, Ramiro

Porto Alegre 1999

Schwartzman, Simon 1979

Serra Costa, J. J. da 1972

Teixeira, Elza

Vieira de Souza

Tiomno, Jayme
'A física no Brasil'. Em Fernando Azevedo (org.), As ciências no Brasil. Rio de Janeiro, Editora UFRJ, vols. 1, 2, pp. 191-232.

Physical-chemistry. $5^{\text {a }}$ ed., Nova York/Londres, John Wiley and Sons/Chapman and Hall Limited, pp. 1-3.

Depoimento. Em Maria L. Fávero (org.), Faculdade Nacional de Filosofia. Rio de Janeiro, Universidade Federal do Rio de Janeiro/Proedes, pp. 289-308.

Dicionário deFísica.

Rio de Janeiro, Nova Fronteira, p. 160.

'O bituary for Sergio Pereira da Silva Porto'. Em H. Walther et al. (org.), Laser spectroscopy IV. Berlim/Heidelberg/Nova York, Springer-Verlag, p. VII.

'A física no Brasil'. Em Mário Guimarães Ferri et alii (org.), História dasciências no Brasil. São Paulo, EPU/Editora da Universidade de São Paulo, vol.1, pp. 61-92.

'Jacques Danon'. Arquivo Jacques Danon: in ventário sumário. Rio de Janeiro, Museu de Astronomia e Ciências Afins, pp. 7-10.

Formação da comunidade científica no Brasil. São Paulo/Rio de Janeiro, Companhia Editora Nacional/Finep.

Carneiro Felippe: estudo biográfico.

Rio de Janeiro, CNEN, 38p.

Depoimento. Em Fávero, op. cit., pp. 166-79.

Depoimento. Em Fávero, op. cit., pp. 255-74. 